PREPARED FOR THE U.S. DEPARTMENT OF ENERGY, UNDER CONTRACT DE-AC02-76-CHO-3073

PPPL-3101

UC-427

GYROKINETIC SIMULATION OF INTERNAL KINK MODES

BY

H. NAITOU, K. TSUDA, W. LEE AND R.D. SYDORA

MAY 1995
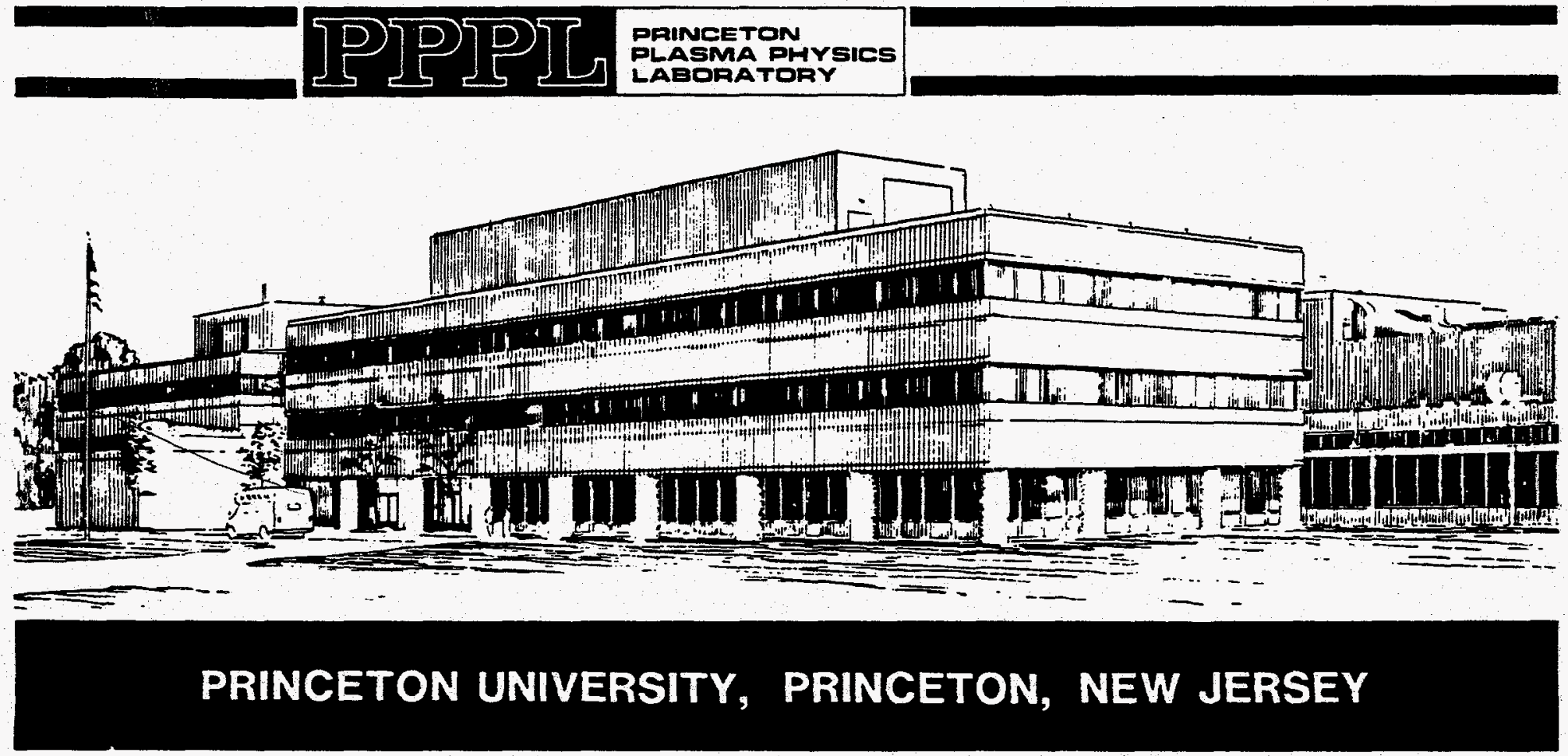


\section{NOTICE}

This report was prepared as an account of work sponsored by an agency of the United States Government. Neither the United States Government nor any agency thereof, nor any of their employees, makes any warranty, express or implied, or assumes any legal liability or responsibility for the accuracy, completeness, or usefulness of any information, apparatus, product, or process disclosed, or represents that its use would not infringe privately owned rights. Reference herein to any specific commercial produce, process, or service by trade name, trademark, manufacturer, or otherwise, does not necessarily constitute or imply its endorsement, recommendation, or favoring by the United States Government or any agency thereof. The views and opinions of authors expressed herein do not necessarily state or reflect those of the United States Government or any agency thereof.

\section{NOTICE}

This report has been reproduced from the best available copy. Available in paper copy and microfiche.

Number of pages in this report: 49

DOE and DOE contractors can obtain copies of this report from:

Office of Scientific and Technical Information

P.O. Box 62

Oak Ridge, TN 37831;

(615) 576-8401.

This report is publicly available from the:

National Technical Information Service

Department of Commerce

5285 Port Royal Road

Springfield, Virginia 22161

(703) $487-4650$ 


\section{DISCLAIMER}

Portions of this document may be illegible in electronic image products. Images are produced from the best available original document. 


\title{
Gyrokinetic Simulation of Internal Kink Modes
}

\author{
Hiroshi Naitou and Kenji Tsuda \\ Department of Electrical and Electronical Engineering \\ Yamaguchi University, Tokiwadai 2557, Ube 755, Japan \\ W. W. Lee \\ Princeton Plasma Physics Laboratory, Princeton University \\ P. O. Box 451, Princeton, New Jersey 08543 \\ R. D. Sydora
}

Department of Physics, University of California at Los Angeles

Los Angeles, California 90024

1 


\section{Abstract}

Internal disruption in a tokamak has been simulated using a three-dimensional magnetoinductive gyrokinetic particle code. The code operates in both the standard gyrokinetic mode (total- $f$ code) and the fully nonlinear characteristic mode ( $\delta f$ code). The latter, a recent addition, is a quiet low noise algorithm. The computational model represents a straight tokamak with periodic boundary conditions in the toroidal direction. The plasma is initially uniformly distributed in a square cross section with perfectly conducting walls. The linear mode structure of an unstable $m=1$ (poloidal) and $n=1$ (toroidal) kinetic internal kink mode is clearly observed, especially in the $\delta f$ code. The width of the current layer around the $x$-point, where magnetic reconnection occurs, is found to be close to the collisionless electron skin depth. This is consistent with the theory in which electron inertia has a dominant role. The nonlinear behavior of the mode is found to be quite similar for both codes. Full reconnection in the Alfven time scale is observed along with the electrostatic potential structures created during the full reconnection phase. The $E \times B$ drift due to this electrostatic potential dominates the nonlinear phase of the development after the full reconnecton. 


\section{Introduction}

Gyrokinetic particle simulation ${ }^{1-4}$ is generally considered to be a useful tool for simulating the low frequency microinstabilities in a tokamak plasma. Since the high frequency phenomena are eliminated in the gyrokinetic formulation by averaging over the gyro-orbit of individual particles, only the phenomena with frequencies less than the ion gyrofrequency are included in the system of gyrokinetic equations. Therefore, we can simulate the low frequency phenomena by using an explicit time integrating algorithm with large time steps. Also, we can use considerably larger grid size compared with a standard particle code in which spatial grid size must be equal to or less than the electron Debye length in order not to excite unphysical aliasing modes. ${ }^{5}$ These properties make it possible to incorporate full tokamak geometry into the code to simulate realistic transport phenomena in tokamaks. This is the approach taken by the "Numerical Tokamak Project" ${ }^{6-8}$ for the purpose of identifying the key physics issues associated with "anomalous" transport in tokamaks. For example, drift-wave fluctuations driven unstable by trapped particles ${ }^{9}$ and ion temperature gradient drift instability ${ }^{10}$ are simulated for full tokamak geometry with gyrokinetic particle codes.

Although the thermal noise level of gyrokinetic particle simulation is far lower than the standard particle code, ${ }^{11}$ we still need more than $10^{6-8}$ particles to simulate a full torus. This is because the saturation level for the important modes are usually not much 
higher than the thermal noise level which, in curn, is inversely proportional to the square root of the number of particles used in the simulation. Recently there has been an innovation in particle simulation techniques, which drastically reduces the thermal noise level due to the discreteness of individual particles. It is called the $\delta f$ method and it was first independently proposed by Dimits ${ }^{12}$ and Kotschenruether. ${ }^{13}$ In the standard particle simulation, the Klimontovich equation is solved by following the particle orbit with constant weights (charge and mass) under the influence of the self-consistent fields. For the new $\delta f$ method, the deviation $\delta f$ from the equilibrium distribution function $f_{0}$ is simulated by following the characteristics of the particles with variable weights. Detailed accounts of the methods for the partially linearized and fully nonlinear gyrokinetic equations are given by Refs. 14 and 15, respectively. The application to the three-dimensional toroidal system can be found in Ref. 10 .

Because the nonlinear $E \times B$ advection associated with the microinstabilities is believed to be chiefly responsible for the anomalous transport in tokamaks, the use of gyrokinetic particle codes mentioned above has so far been limited to electrostatic simulations. However, there is another class of modes in tokamaks such as the MHD modes with kinetic modification that have yet to be explored by gyrokinetic particle codes. The resonant particle destabilization of internal kink modes ("fishbones"), ${ }^{16}$ toroidicity induced Alfven eigenmodes (TAE), ${ }^{17}$ the stabilization of sawteeth in the case of auxiliary heating ("monster sawteeth") ${ }^{18}$ sawtooth collapse on the fast time scale,${ }^{19}$ the physics of $q$ (safety factor) $<1$ on axis after the sawtooth crash $^{20-22}$ are some of the examples. The phenomena asso- 
ciated with all these modes can not be simply explained by the conventional MHD theory or the standard MHD simulation. Although fluid simulation codes that include kinetic effects may also be used, we believe that the gyrokinetic particle approach, which is free of the closure problems, is better suited for these types of global simulation.

The sawtooth collapse in tokamaks is not a very well understood subject. For example, some of the existing theories, ${ }^{23,24}$ based on the nonlinear development of the $m=1$ (poloidal) and $n=1$ (toroidal) internal kink mode, predict a full reconnection process in which original hot core region is pushed out and disappears while a $m=1$ island grows and fill out the $q<1$ region. Thus, the original equilibrium with the central safety factor $q_{0}$ less than unity is transformed to a state with $q_{0} \simeq 1$. For the treatment of the physics inside the current layer where magnetic reconnection occurs, Kadomtsev assumes electron collisions ${ }^{23}$ while Wesson uses electron inertia. ${ }^{24}$ Also, the importance of the electron inertial effect for the collisionless reconnection have been recognized in astrophysics. ${ }^{25}$ Since these predictions are still controversial, in this paper, we intend to address some of these physics questions by simulating the $m=1$ and $n=1$ kinetic internal kink mode in a straight tokamak model using a modified version of the three-dimensional magnetoinductive gyrokinetic particle code. ${ }^{8}$ The code is based on the nonlinear gyrokinetic formalism developed by Hahm et al. ${ }^{2}$ for finite- $\beta$ plasmas. For simplicity, we have limited ourselves to the straight tokamak model without the toroidal effects, and also neglected the finite Larmor radius effects for the ions $\left(\rho_{i}=0\right)$. Thus, the ions are treated as drift kinetic particles just as the electrons. The ion polarization density response is now 
included in the gyrokinetic Poisson equation. Moreover, we do not include the physics of energetic ions which may have strong influence on the development of the $m=1$ and $n=1$ mode. However, even with these simplifications, nonlinear physics associated with kinetic internal kink modes is still needed to be resolved. We expect that the inclusion of the full dynamics of drift kinetic electrons will have a crucial effect on the kinetic modification of the $m=1$ and $n=1$ modes. The simulation results (especially reconnection time and the current layer width around the reconnection point) will be compared with the theory given by Wesson, ${ }^{24}$ which predicts that the first stage of the sawtooth crash operates on the Alfven time scale and the width of the current layer is of the order of the collisionless electron skin depth $c / \omega_{\text {pe }}$, where $c$ is the speed of light in vacuum and $\omega_{p e}$ is the electron plasma frequency. In addition, this paper is intended to make two important points. The first one is to demonstrate that gyrokinetic particle simualtion can indeed simulate low-frequency MHD modes. The second point is to show the usefulness of the nonlinear characteristic method ( $\delta f$ method) in the three-dimensional magneto-inductive gyrokinetic particle code. This aspect is especially important when the instability is very weak and the mode amplitude is very small in the linear phase of the instability.

Very recently, it has been pointed out that there can be a state with $q_{0}$ less than unity after the sawtooth collapse. ${ }^{26}$ Biskamp and Drake ${ }^{27}$ have demonstrated that there is a second stage following the first full reconnection phase. In this second stage, a reconnection prccess takes place driven by the strong flows generated during the first 
reconnection phase. As a result, a region with $q_{0}<1$ is re-established. Our simulation results show the similar trend, i.e., the central safety factor goes down to 0.95 after the reconnection, whereas the original central safety facter is 0.85 before the reconnection.

The outline of the paper is as follows. The basic formulation of the gyrokinetic model is summarized in Sec. 2. The $\delta f$ method is described in Sec. 3. Sections 4 and 5 present the simulation studies of the linear and nonlinear phenomena of the kinetic internal kink mode (sawtooth collapse), using the standard gyrokinetic (total- $f$ ) code and the $\delta f$ code, respectively. The result for the re-organization of the $q_{0}<1$ region after the full reconnection phase is also presented in Sec. 5. The conclusions and discussions are given in Sec. 6.

\section{Gyrokinetic Formulation}

Let us first summarize the gyrokinetic formulation used for the three-dimensional kinetic internal kink mode simulation. The basic equations are based on the formulation given by Hahm et al.. ${ }^{2}$ To the lowest order in the long wavelength limit, the gyrokinetic equations are derived using the ordering of

$$
\frac{\omega}{\omega_{c i}} \simeq \frac{\rho_{t i}}{L} \simeq \frac{k_{\|}}{k_{\perp}} \simeq \frac{e \phi}{T_{e}} \simeq \frac{\delta B}{B} \simeq O(\epsilon), \quad k_{\perp} \rho_{t i} \simeq O(1)
$$

where $\omega$ is a characteristic frequency of a mode, $\omega_{c i}$ is a ion cyclotron frequency, $\rho_{t i}$ is a thermal ion gyroradius, $L$ is a characteristic length of the gradient of macroscopic quanti- 
ties like density, temperature, or magnetic field, $k_{\|}$and $k_{\perp}$ are wave numbers parallel and perpendicular to the magnetic field, respectively, $\phi$ is a potential, $T_{e}$ is a electron temperature, $\delta B$ is the variation of the magnetic field from a constant longitudinal magnetic field $B_{z}$, and $\epsilon$ is the smallness parameter. This ordering is valid for the low-frequency phenomena in the bulk plasma in tokamaks. Electrons are treated in the drift kinetic approximation. In this paper, we also neglect the finite gyroradius effect of the ions, i.e., $k_{\perp} \rho_{t i} \simeq 0$, because we want to simulate large scale MHD modes. There is a possibility that ion gyroradius effects may play a important role especially in the region of magnetic reconnection. Such an effect is outside of the scope of the present paper.

The gyrokinetic simulation reported in this paper has been carried out in a straight tokamak model in the $x, y, z$ coordinates with a rectangular box of $L_{x}, L_{y}$, and $L_{z}$. The box is surrounded by the perfectly conducting walls in the $x$ and $y$ (poloidal) directions and, in the $z$ (toroidal) direction, periodic boundary conditions are assumed. The box is uniformly filled with plasma particles initially and a strong and constant magnetic field is applied in the $z$ direction, $B=B_{0} b$. We only keep the field line bending terms in the equations of motion and the compressional effects on the toroidal magnetic field is neglected in the low-beta approximation. The poloidal magnetic fields,

$$
B_{x}=\frac{\partial A_{z}}{\partial y}, B_{y}=-\frac{\partial A_{z}}{\partial x}
$$

are produced by the current in the $z$-direction, where $A_{z}$ is the $z$ component of the vector potential. The electrostatic electric field $\boldsymbol{E}_{L}$ is given by the gradient of the electrostatic 
potential,

$$
E_{L}=-\nabla \phi
$$

The bounded boundary conditions are used for $\phi$ and $A_{z}$, where they are set to zero at the walls. There is also a inductive electric field in the $z$ direction, $E_{T z}\left(=-\partial A_{z} / \partial t\right)$.

The guiding center position $\boldsymbol{x}_{j}^{\sigma}$ and the velocity $v_{z j}^{\sigma}$ along a magnetic field for $j$-th particle is advanced in time with the self-consistent electromagnetic fields produced by the particles themselves, where the superscript $\sigma$ represents the species. It should be noted that, in the gyrokinetic ordering, the parallel velocity is always in the direction parallel to the local magnetic field and so is $E_{T z}$, the induction electric field. $E_{L}$, the electrostatic field, gives rise to the $E \times B$ drift across the external magnetic field for the particles as well as their parallel acceleration along the local magnetic field. Thus, the equations of motion for the $j$-th particle are given $b^{2}$

$$
\frac{d \boldsymbol{x}_{j}^{\sigma}}{d t}=v_{z j}^{\sigma} \boldsymbol{b}^{*}+\frac{E_{L} \times \boldsymbol{b}}{B_{0}}
$$

and

$$
\frac{d v_{z j}^{\sigma}}{d t}=\frac{q_{\sigma}}{m_{\sigma}}\left(\boldsymbol{E}_{L} \cdot \boldsymbol{b}^{*}+E_{T z}\right)
$$

where $m_{\sigma}$ is the mass, $q_{\sigma}$ is the charge, and $b^{*}$ is the unit vector along the magnetic field,

$$
\boldsymbol{b}^{*}=\boldsymbol{b}+\frac{\nabla A_{z} \times \boldsymbol{b}}{B_{0}}
$$

The calculation of the induction electric field is the most complicated part in a magnetoinductive code. However $E_{T z}$ does not appear explicitly in the system of equations if the 
angular momentum $p_{z}$ is used. ${ }^{2}$ More specifically, if we use

$$
p_{z j}^{\sigma}=v_{z j}^{\sigma}+\frac{q_{\sigma}}{m_{\sigma}} A_{z}\left(\boldsymbol{x}_{j}^{\sigma}, t\right)
$$

to replace the velocity $v_{z j}$ and also introduce a generalized potential given by

$$
\Psi\left(\boldsymbol{x}_{j}^{\sigma}, t\right)=\phi\left(\boldsymbol{x}_{j}^{\sigma}, t\right)-v_{z j}^{\sigma} A_{z}\left(\boldsymbol{x}_{j}^{\sigma}, t\right)
$$

the equations of motion given by (4) and (5) can be replaced by the following equations:

$$
\begin{gathered}
\frac{d \boldsymbol{x}_{j}^{\sigma}}{d t}=v_{z j}^{\sigma} \boldsymbol{b}-\frac{\nabla \Psi \times \boldsymbol{b}}{B_{0}} \\
\frac{d p_{z j}^{\sigma}}{d t}=-\frac{q_{\sigma}}{m_{\sigma}} \boldsymbol{b} \cdot \nabla \Psi
\end{gathered}
$$

The electrostatic potential $\phi$ is determined by the gyrokinetic Poisson equation,

$$
\nabla^{2} \phi+\frac{\omega_{p i}^{2}}{\omega_{c i}^{2}} \nabla_{\perp}^{2} \phi=\frac{e}{\epsilon_{0}} \sum_{j} \delta\left(\boldsymbol{x}-\boldsymbol{x}_{j}^{e}\right)-\frac{q_{i}}{\epsilon_{0}} \sum_{j} \delta\left(\boldsymbol{x}-\boldsymbol{x}_{j}^{i}\right)
$$

where $\epsilon_{0}$ is the permitivity of vacuum, $\omega_{p i}$ is the ion plasma frequency, $\delta$ is the delta function, and $\nabla_{\perp}$ is the gradient operator perpendicular to the longitudinal magnetic field, and $q_{e}=-e$. The second term in the left-hand-side of the above equation represents the ion polarization shielding effects, which usually dominates the first (Debye) term. In the $p_{z}$ formulation, the calculation of $A_{z}$ is somewhat complicated because we can not use $v_{z}$ to calculate the current density. The Ampere's law in the $p_{z}$ formulation becomes

$$
\begin{aligned}
\left(\nabla_{\perp}^{2}-\frac{\omega_{p e}^{2}+\omega_{p i}^{2}}{c^{2}}\right) A_{z} & =\mu_{0} e \sum_{j} p_{z j}^{e} \delta\left(\boldsymbol{x}-\boldsymbol{x}_{j}^{e}\right)+\frac{\omega_{p e}^{2}}{c^{2}} A_{z}\left[\frac{\sum_{j} \delta\left(\boldsymbol{x}-\boldsymbol{x}_{j}^{e}\right)}{n_{0 e}}-1\right] \\
& -\mu_{0} q_{i} \sum_{j} p_{z j}^{i} \delta\left(\boldsymbol{x}-\boldsymbol{x}_{j}^{i}\right)+\frac{\omega_{p i}^{2}}{c^{2}} A_{z}\left[\frac{\sum_{j} \delta\left(\boldsymbol{x}-\boldsymbol{x}_{j}^{i}\right)}{n_{0 i}}-1\right]
\end{aligned}
$$


where $\mu_{0}$ is the vacuum permeability, $n_{0 \sigma}$ is the equilibrium number density $\left(e n_{0 e}=q_{i} n_{0 i}\right)$. The particular form of the equation, where the dominant terms are moved to left-handside, is to facilitate the iterative scheme for calculating $A_{z}$ using the usual fast Fourier transformation technique, where we utilize the $A_{z}$ obtained in the previous step as the initial guess. Note that once $A_{z}$ is obtained in each time step we can obtain $v_{z j}^{\sigma}$ from $p_{z j}^{\sigma}$. Eqs. (9) to (12) form a self-consistent set of equations in which the total energy $\mathcal{E}_{T}$ conserves. The energy conservation for the simulation system is given by

$$
\begin{aligned}
\mathcal{E}_{T} & =\mathcal{E}_{K}+\mathcal{E}_{E}+\mathcal{E}_{B} \\
\mathcal{E}_{K} & =\sum_{j} \frac{1}{2} m_{e}\left[p_{z j}^{e}+\frac{e}{m_{e}} A_{z}\left(\boldsymbol{x}_{j}^{e}, t\right)\right]^{2}+\sum_{j} \frac{1}{2} m_{i}\left[p_{z j}^{i}-\frac{q_{i}}{m_{i}} A_{z}\left(\boldsymbol{x}_{j}^{i}, t\right)\right]^{2}, \\
\mathcal{E}_{E} & =\frac{1}{2} \epsilon_{0} \sum_{\boldsymbol{k}} k^{2}\left|\phi_{k}\right|^{2}+\frac{1}{2} \epsilon_{0} \frac{\omega_{p i}^{2}}{\omega_{c i}^{2}} \sum_{\boldsymbol{k}} k_{\perp}^{2}\left|\phi_{k}\right|^{2} \\
\mathcal{E}_{B} & =\frac{1}{2} \sum_{\boldsymbol{k}} k_{\perp}^{2}\left|A_{z k}\right|^{2}
\end{aligned}
$$

where $\mathcal{E}_{K}$ is the kinetic energy of particles, $\mathcal{E}_{E}$ is the electric field energy, which is the sum of the conventional electric field energy and the ion sloshing energy, and $\mathcal{E}_{B}$ is a magnetic field energy. The ion sloshing energy is identical to the fluid kinetic energy in the MHD terminology. The first term on the left hand side of Eq.(15) is negligible, hence $\mathcal{E}_{E}$ is essentially the fluid kinetic energy. 


\section{Formulation of $\delta f$ Method}

In general, the kinetic equation we are solving in the particle simulation is of the Klimontovich type, where the phase space distribution is represented by individual particles. Therfore, it is natural that the thermal noise level is determined by the number of particles used in the simulation. This noise level, which is associated with the fluctuating

field energy can be estimated by the fluctuation dissipation theorem. ${ }^{11}$ This is indeed the case when we solve the gyrokinetic Vlasov equation,

$$
\frac{d f_{\sigma}}{d t}=\frac{\partial f_{\sigma}}{\partial t}+\left(v_{z} \boldsymbol{b}-\frac{\nabla \Psi \times \boldsymbol{b}}{B_{0}}\right) \cdot \frac{\partial f_{\sigma}}{\partial \boldsymbol{x}}-\frac{q_{\sigma}}{m_{\sigma}} \boldsymbol{b} \cdot \nabla \Psi \frac{\partial f_{\sigma}}{\partial p_{z}}=0
$$

using the total $f$ scheme. ${ }^{1}$ This scheme can be viewd as a sampling technique for solving the equation, for which markers in the phase space are used to represent particle trajectories evolving in time according to the self-consistent fields. On the other hand, the $\delta f$ method uses the marker only to account for the deviation from a prescribed equilibrium and, therefore, is noise free at $t=0$. To do so, we have to first decompose the distribution function into the equilibrium distribution $f_{0 \sigma}$ and the perturbed distribution $\delta f_{\sigma}$,

$$
f_{\sigma}\left(\boldsymbol{x}, p_{\boldsymbol{z}}, t\right)=f_{0 \sigma}\left(x, y, p_{z}\right)+\delta f_{\sigma}\left(\boldsymbol{x}, p_{z}, t\right)
$$

where we have assumed only $x$ and $y$ dependences for $f_{0 \sigma}$, i.e., $z$ is an ignorable coordinate for the equilibrium quantities. To represent the perturbation, we introduce a weight $w_{j}^{\sigma}$ for each marker. Although each marker still retain the same charge, $q_{\sigma}$, and the same 
mass, $m_{\sigma}$, the perturbed distribution $\delta f_{\sigma}$ can now be expressed in terms of $w_{j}^{\sigma}$ 's as

$$
\delta f_{\sigma}\left(\boldsymbol{x}, p_{z}, t\right)=\sum_{j} w_{j}^{\sigma}(t) \delta\left[\boldsymbol{x}-\boldsymbol{x}_{j}^{\sigma}(t)\right] \delta\left[p_{z}-p_{z j}^{\sigma}(t)\right] .
$$

The time dependence of $w_{j}^{\sigma}$ is given by Parker and Lee ${ }^{15}$ as

$$
\frac{d w_{j}^{\sigma}}{d t}=-\left.\left(1-w_{j}^{\sigma}\right)\left(\frac{d \boldsymbol{x}_{j}^{\sigma}}{d t} \cdot \frac{\partial f_{0 \sigma}}{\partial \boldsymbol{x}}+\frac{d p_{z j}^{\sigma}}{d t} \frac{\partial f_{0 \sigma}}{\partial p}\right) \frac{1}{f_{0 \sigma}}\right|_{\boldsymbol{x}=\boldsymbol{x}_{j}^{\sigma}, p_{z}=p_{z j}^{\sigma}},
$$

where the initial weights should be $\left|w_{j}^{\sigma}(t=0)\right| \ll 1$ and the initial distribution is given by the markers as,

$$
f_{0 \sigma}=f_{\sigma}\left(x, y, p_{z}, t=0\right)=\sum_{j} \delta\left[x-x_{j}^{\sigma}(t=0)\right] \delta\left[p_{z}-p_{z j}^{\sigma}(t=0)\right] .
$$

For the electron equilibrium distribution $f_{0 e}$, we have assumed a shifted Maxwellia.ı distribution with a drift velocity of $v_{D}(x, y)$ in the direction of the external magnetic field,

$$
f_{0 e}=\frac{n_{0 e}}{\sqrt{2 \pi} v_{t e}} \exp \left\{-\frac{\left[p_{z}^{e}+\frac{e}{m_{e}} A_{z 0}(x, y)-v_{D}(x, y)\right]^{2}}{2 v_{t e}^{2}}\right\}
$$

where $v_{t e}$ is the electron thermal speed. As for the ion equilibrium distribution $f_{0 i}$, a Maxwellian distribution without a drift velocity is assumed,

$$
f_{0 i}=\frac{n_{0 i}}{\sqrt{2 \pi} v_{t i}} \exp \left\{-\frac{\left[p_{z}^{i}-\frac{g_{i}}{m_{i}} A_{z 0}(x, y)\right]^{2}}{2 v_{t i}^{2}}\right\}
$$

where $v_{t i}$ is an ion thermal speed. $A_{z 0}$ is the initial equilibrium $A_{z}$ obtained from

$$
\nabla_{\perp}^{2} A_{z 0}(x, y)=\mu_{0} e n_{0 e} v_{D}(x, y)
$$

Using the equillibrium distributions given by Eqs. (22) and (23), Eq. (20) becomes

$$
\begin{aligned}
\frac{d w_{j}^{e}}{d t}= & -\left(1-w_{j}^{e}\right) \frac{p_{z j}^{e}+\frac{e}{m_{e}} A_{z 0}-v_{D}}{v_{t e}^{2}} \\
& {\left[\frac{d x_{j}^{e}}{d t}\left(-\frac{e}{m_{e}} \frac{\partial A_{z 0}}{\partial x}+\frac{\partial v_{D}}{\partial x}\right)+\frac{d y_{j}^{e}}{d t}\left(-\frac{e}{m_{e}} \frac{\partial A_{z 0}}{\partial y}+\frac{\partial v_{D}}{\partial y}\right)-\frac{d p_{z j}^{e}}{d t}\right], }
\end{aligned}
$$


and

$$
\frac{d w_{j}^{i}}{d t}=-\left(1-w_{j}^{i}\right) \frac{p_{z j}^{i}-\frac{q_{i}}{m_{i}} A_{z 0}}{v_{t i}^{2}}\left(\frac{d x_{j}^{i}}{d t} \frac{q_{i}}{m_{i}} \frac{\partial A_{z 0}}{\partial x}+\frac{d y_{j}^{i}}{d t} \frac{q_{i}}{m_{i}} \frac{\partial A_{z 0}}{\partial y}-\frac{d p_{z j}^{i}}{d t}\right)
$$

With the weighted charge density as the source term, the gyrokinetic Poisson's equation becomes

$$
\nabla^{2} \phi+\frac{\omega_{p i}^{2}}{\omega_{c i}^{2}} \nabla_{\perp}^{2} \phi=\frac{e}{\epsilon_{0}} \sum_{j} w_{j}^{e} \delta\left(\boldsymbol{x}-\boldsymbol{x}_{j}^{e}\right)-\frac{q_{i}}{\epsilon_{0}} \sum_{j} w_{j}^{i} \delta\left(\boldsymbol{x}-\boldsymbol{x}_{j}^{i}\right) .
$$

The corresponding Ampere's law can then be written as

$$
\begin{aligned}
\nabla_{\perp}^{2} A_{z}(\boldsymbol{x}, t) & =-\mu_{0} \sum_{\sigma=e, i} q_{\sigma} \int\left[p_{z}-\frac{q_{\sigma}}{m_{\sigma}} A_{z}(\boldsymbol{x}, t)\right] f_{0 \sigma}\left(x, y, p_{z}, t\right) d p_{z} \\
& -\mu_{0} \sum_{\sigma=e, i} q_{\sigma} \sum_{j}\left[p_{j}^{\sigma}-\frac{q_{\sigma}}{m_{\sigma}} A_{z}\left(\boldsymbol{x}_{j}^{\sigma}, t\right)\right] w_{j}^{\sigma} \delta\left(\boldsymbol{x}-\boldsymbol{x}_{j}^{\sigma}\right)
\end{aligned}
$$

By defining $\delta A_{z}=A_{z}-A_{z o}$, we have the following equation,

$$
\begin{aligned}
& \left(\nabla_{\perp}^{2}-\frac{\omega_{p e}^{2}+\omega_{p i}^{2}}{c^{2}}\right) \delta A_{z} \\
= & \mu_{0} e \sum_{j} p_{z j}^{e} w_{j}^{e} \delta\left(\boldsymbol{x}-\boldsymbol{x}_{j}^{e}\right)+\frac{\omega_{p e}^{2}}{c^{2} n_{0 e}}\left(A_{z 0}+\delta A_{z}\right) \sum_{j} w_{j}^{e} \delta\left(\boldsymbol{x}-\boldsymbol{x}_{j}^{e}\right) \\
- & \mu_{0} q_{i} \sum_{j} p_{z j}^{i} w_{j}^{i} \delta\left(\boldsymbol{x}-\boldsymbol{x}_{j}^{i}\right)+\frac{\omega_{p i}^{2}}{c^{2} n_{0 i}}\left(A_{z 0}+\delta A_{z}\right) \sum_{j} w_{j}^{i} \delta\left(\boldsymbol{x}-\boldsymbol{x}_{j}^{i}\right)
\end{aligned}
$$

which can then be solved iteratively.

The calculations for the field energies, $\mathcal{E}_{E}$ and $\mathcal{E}_{B}$, are the same as the total- $f$ case and are given by Eqs. (15) and (16), respectively. Kinetic energy $\mathcal{E}_{K}$ in the $\delta f$ formulation becomes,

$$
\mathcal{E}_{K}=\frac{1}{2} m_{e} v_{t e}^{2} N_{e}+\frac{1}{2} m_{e} n_{0 e} \int\left(\frac{e}{m_{e}} \delta A_{z}+v_{D}\right)^{2} d x
$$




$$
\begin{aligned}
& +\frac{1}{2} m_{e} \sum_{j}\left[p_{j}^{e}+\frac{e}{m_{e}} A_{z}\left(\boldsymbol{x}_{j}^{e}, t\right)\right]^{2} w_{j}^{e} \\
& +\frac{1}{2} m_{i} v_{t i}^{2} N_{i}+\frac{1}{2} m_{i} n_{0 i} \int\left(\frac{q_{i}}{m_{i}} \delta A_{z}\right)^{2} d \boldsymbol{x} \\
& +\frac{1}{2} m_{i} \sum_{j}\left[p_{j}^{i}-\frac{q_{i}}{m_{i}} A_{z}\left(\boldsymbol{x}_{j}^{i}, t\right)\right]^{2} w_{j}^{i},
\end{aligned}
$$

where $N_{e}$ and $N_{i}$ are the total numbers of electrons and ions, respectively. We can also calculate the kinetic energy by using the markers as given by Eq. (14). It is to be noted that the conservation properties for the $\delta f$ method improves with the number of particles used and the success of the $\delta f$ method depends on how well the assembly of markers representing the velocity distribution function. In general, the random (thermal) start used in the usual particle simulation works well for the $\delta f$ method. However, the quiet start technique using the bit-reversed numbers ${ }^{29}$ for determining positions of particles in the phase space is best suited for the $\delta f$ simulation.

\section{Simulation Results of Total- $f$ Code}

Let us first show the simulation results using the total- $f$ code for simulating the kinetic internal kink mode in a straight tokamak with a square cross section. The dimensions for the simulation system are $L_{x} \times L_{y} \times L_{z}=64 \Delta_{\perp} \times 64 \Delta_{\perp} \times 32 \Delta_{\|}$, where $\Delta_{\perp}$ and $\Delta_{\|}$are the grid sizes perpendicular and parallel to the longitudinal magnetic field,

respectively and $\Delta_{\|}$is a factor of 1000 longer than $\Delta_{\perp}$. The total number of particles used in the simulation for each species is $N=8,388,608$. As for the equilibrium current 
density $J_{z 0}$, we have used the profile given by Strauss, ${ }^{28}$

$$
J_{z 0}(x, y)=\frac{1}{\mu_{0}}\left[\left(\frac{\pi}{L_{x}}\right)^{2}+\left(\frac{\pi}{L_{y}}\right)^{2}\right] A_{0} \sin \left(\frac{\pi x}{L_{x}}\right) \sin \left(\frac{\pi y}{L_{y}}\right)
$$

The corresponding $A_{z 0}$, and $q$ profiles for this current density is

$$
\begin{gathered}
A_{z 0}(x, y)=A_{0} \sin \left(\frac{\pi x}{L_{x}}\right) \sin \left(\frac{\pi y}{L_{y}}\right), \\
q=\frac{4 L_{x} L_{y} B_{0}}{\pi^{2} A_{0} L_{z}} K\left(\sin ^{2} \psi\right), \quad \psi=\frac{A_{0}-A_{z}}{A_{0}},
\end{gathered}
$$

where $K$ is an elliptic integral of the first kind. The central $q$ value is chosen to be 0.85 in the simulation. Thus, this equilibrium $q$ profile is generated by the electron current in the $z$ direction. The drift velocity for the electrons in the center is $0.32 v_{t e}$ which is small enough without exciting the velocity space instability artificially. Only the modes with $n=0$ and $n= \pm 1$ were kept in the simulation, since retaining the higher $n$ modes would require a significant reduction in the time step, $\Delta t$, used in the simulation because of the $k_{\|} v_{t e} \Delta t<1$ restriction. ${ }^{1}$ The significance of this approximation will be discussed in the next section. The initial velocity distribution functions given by Eqs. (22) and (23) are generated by using the bit-reversed numbers. ${ }^{29}$ However, the difference of the results between using the quiet start and the thermal start techniques in the total- $f$ code is quite small, although the former gives a slightly better noise property initially.

The other simulation parameters are: $\omega_{c e} / \omega_{p e}=1, c / \omega_{p e}=4 \Delta_{\perp}, v_{t e} / v_{A}=0.25$, $\Delta_{\perp}=\rho_{s i}=\sqrt{T_{e} / m_{i}} / \omega_{c i}, m_{i} / m_{e}=1836, T_{i} / T_{e}=1, \beta=\left(v_{t e} / v_{A}\right)^{2}\left(m_{e} / m_{i}\right)=0.000034$ $\Delta t=1.743 \omega_{c i}^{-1}=0.00932 L_{z} / v_{A}$, where $v_{A}=c\left(\omega_{c i} / \omega_{p i}\right)$ is the Alfven velocity, and the 
corresponding Alfven time is $\tau_{A}=L_{z} / v_{A}$. The finite size Gaussian particles are used, where the particle sizes are $a_{x}=\Delta_{\perp}, a_{y}=\Delta_{\perp}$, and $a_{z}=\Delta_{\|}$in $x, y$, and $z$ directions, respectively. The number of total time steps is 2200 . The total CPU time was 23.8 hours on the $\mathrm{SX}-3 / 24 \mathrm{R}$ vector/parallel supercomputer by NEC with $2 \mathrm{CPU}$ units. (However, the simulation has been carried out using only one processor.)

The time evolution for the various energies, defined in Eqs. (13)-(16), are shown in Fig.1, where only the deviations from their respective initial values normalized by the initial total energy $\mathcal{E}_{T 0}$ are plotted. In the growth phase, magnetic field energy $\mathcal{E}_{B}$ decreases in time, while the ion sloshing (fluid kinetic) energy $\mathcal{E}_{E}$ and $\mathcal{E}_{K}$ (mainly from the parallel electron kinetic energy) increase rapidly. The parallel kinetic energy of the ions does not change throughout the simulation. Thus, the magnetic field energy is released to the fluid kinetic energy and the electron kinetic energy as a result of the magnetic reconnection. We will call this time period, before $\mathcal{E}_{E}$ reaches maximum and $\mathcal{E}_{B}$ becomes minimum, the full reconnection phase. After the full reconnection phase, $\mathcal{E}_{E}$ starts to decrease and $\mathcal{E}_{B}$ begins to increase, while $\mathcal{E}_{K}$ stays more or less unchanged in time. Hence, one may conclude that the energy that went to the fluid kinetic energy in the full reconnection phase is now reverted back to the magnetic field energy.

The energy conservation is fairly good and the deviation at the end of the simulation is less than $7 \times 10^{-5}$ of the total energy. We believe this small deviation is due to the poor resolution of the plasma response associated with the short wavelength oscillations. A smaller time step in the simulation can improve the conservation property. However, 
we do not expect that an improvement in energy cnservation would have any effect on the reconnection physics.

The time evolution of the magnetic field structure at the cross section $z=0$ is depicted in Fig.2. The Roman numerals in the figure correspond to the different stages of the development shown in Fig.1. We followed the 14 magnetic field lines and plotted them when they moved across the $z=0$ surface. The starting points for the traces of magnetic field lines are equally spaced and are symmetric with respect to the center of the plasma. Here, at $t=0$, each of the magnetic surface actually is consisted of two field lines. The position of the $q=1$ magnetic surface is clearly distinguished by the position of the magnetic island at time I. The outermost three magnetic surfaces are outside the $q=1$ surface, and we can see no change in them from time I to III. However, from time IV to VI, only the outermost two magnetic surfaces are unchanged. At the $q=1$ surface, we can see the x-point where magnetic reconnection occurs due to the motion of core plasma moving toward the wall. The width of the magnetic island increases in time and the core plasma is pushed to the $\mathrm{x}$-point and finally disappears. The process is quite similar to the Kadomtsev and/or Wesson full reconnection process. ${ }^{23,24}$

The contour plots for the electrostatic potentials at different stages of the reconnection process are shown in Fig. 3. (The Roman numerals in the figure correspond to those shown in Fig. 1.) Here, the solid and dotted lines represent the positive and negative potentials, respectively, whereas the bold solid contour is the zero potential. In the early phase of the instability, the amplitude for the coherent mode structure is quite small compared 
with the fluctuations associated with the thermal noise. Therefore, we can clearly see the mode pattern of the kinetic internal kink mode only after the amplitude of the mode is sufficiently large (time IV to VI). (In the next section, we will show that the unstable mode pattern can be clearly seen even in the early phase of instability by using the $\delta f$ method.) Since the direction of the $E \times B$ drift is along the contour of the electrostatic potential and its magnitude is proportinal to the perpendicular gradient, there is a nonvanishing flow to the $x$-point. It is important here to note that the coherent mode pattern of the electrostatic potential maintains its large amplitude even when full reconnection process is almost finished (time VI). After the full reconnection, $q$ is constant and becomes almost unity in the newly formed core region. Hence, in this core region, the mode pattern and magnetic field structure are almost completely synchronized and the variation of the electrostatic potential along the magnetic field is negligibly small. This mode pattern can be expected to persist giving rise to a plasma flow in this shearless region due to the $E \times B$ drift even after the full reconnection phase. This flow will push the peripheral plasma into the central region. Thus, a further reformation of the magnetic field structure can be expected in this phase.

Figure 4 shows the time evolution of the current density $\delta J_{z}=J_{z}-J_{z 0}$ at the $z=$ 0 cross section. $\delta J_{z}$ is normalized by the maximum value of the equilibrium current density. To eliminate the numerical noise, we have averaged $\delta J_{z}$ over a period of $\pm 87 \omega_{c i}^{-1}$. Therefore, $\delta J_{z}$ is approximately the $n=1$ helical component of the current density. We can see the current layer near the $x$-point, along the $q=1$ surface, in which the direction 
of the current is opposite to that of the initial current. The width of the current layer is about $c / \omega_{\text {pe }}$, which is consistent with the theoretical prediction. ${ }^{24}$ The width of the current layer is also displayed in Fig.5, where Fig.5(a) shows the contour plot of $\delta J_{z}$ at $z=0$ at time IV shown in Fig.1, and Fig.5(b) shows $\delta J_{z}$ along the cut $l$, which cross the x-point and the center of the system of Fig.5(a). The current layer width of $c / \omega_{p e}$ is clearly visible.

The growth rate of the instability is calculated from Fig. 6, where the time evolution of the amplitude of the electrostatic potential, defined by $\left(\phi_{\max }-\phi_{\min }\right) / 2$, at the $z=0$ surface is plotted. The measured growth rate is about $\gamma=0.30 \tau_{A}^{-1}$. The full reconnection time of Wesson is given by

$$
\tau=r_{1} \frac{\omega_{p e}}{c} \frac{1}{2 \pi} \frac{1}{1-q_{0}} \tau_{A}
$$

where $r_{1}$ is the radius of the $q=1$ magnetic surface. Since the growth rate is proportional to $\tau^{-1}$, the theoretically predicted growth rate is $\gamma=0.24 \tau_{A}^{-1}$. Hence, the total- $f$ simulation gives good agreement.

\section{Simulation Results of $\delta f$ Code}

Simulations using the $\delta f$ code have been carried out with the same parameters as those for the total- $f$ code except for the number of particles. In this case, we use $N_{e}=N_{i}=524,288$, which is only one sixteenth of the particles used for the total$f$ code. The initial velocity distribution has again been generated by using the bit- 
reversed numbers ${ }^{29}$ and the initial weights are set to be zero for all particles. Therfore, the instability develops from the residue numerical noise. For this set of simulation, the number of time steps is 5500 and the total CPU time is 5.0 hours on the SX-3/24R.

The time evolution for the various quantities associated with the energy conservation [Eqs. (13),(15),(16), and (30)] due to the instability is shown in Fig.7. Compared with the total- $f$ code results (Fig. 1), the conservation properties deteriorate somewhat in this case. This is because of the reduction in the number of particles used in the simulation. Nevertheless, the time history of $\mathcal{E}_{E}$ is almost identical for both cases. The time dependence of $\mathcal{E}_{B}$ is also nearly identical up to the time when it reaches its minimum, and a slight difference is developed afterwards. The major difference is observed in $\mathcal{E}_{K}$. Hence, the relative error in the total energy is $8 \times 10^{-4}$ which is about ten times larger than the total- $f$ case. We believe this difference mainly comes from high energy electrons and has no consequence to the physics. This is consistent with the finding by Parker and Lee ${ }^{15}$ that the conservation of total energy is critically dependent on the number of particles used in the simultion and is difficult to achieve in the $\delta f$ code, but the basic physics trend remains intact. We have indeed observed the tendency that energy conservation improves with the increase in the number of particles used in the simulation, but the physics results remain unchanged. We should also point out that, the $\delta f$ code takes longer time to run because of the low noise level at $t=0$. To remedy the situation, one can introduce a large perturbation at the beginning of the simulation so that the instability can grow to large amplitude in a shorter time. 
The magnetic field structures as a function of time from the $\delta f$ code are shown in Fig.8. They are almost identical to the total- $f$ code results in Fig. 2. However, the reduction of the thermal noise has a dramatic effect on the electrostic potential as shown in Fig.9. Compared with the results from the total- $f$ in Fig. 3, it is striking that we can now see the mode patterns of the unstable kinetic internal kink mode even in the very early stage of the time evolution of the instability. These obviouly are the linear eigenmodes (time I to III ). The time sequence of the snap shots for $\delta J_{z}$ is shown in Fig.10. Again, the low noise feature of the $\delta f$ code is apparent. Namely, only the time evolution of the unstable internal kink mode is depicted in the simulation and the numerical noise is considerably smaller. With such a clear signal, we can see the modification to the linear mode pattern in the nonlinear phase of the development. Fig.11(a) shows the contour plot of $\delta J_{z}$ at $z=0$ at time I specified in Fig.7, and Fig.11(b) gives the amplitude of $\delta J_{z}$ along the cut $l$ of Fig.11(a), which crosses the $x$-point and the center of the system. Again, we see clearly the current layer width of $c / \omega_{p e}$ even when the mode amplitude is quite small. This is the advantage of the $\delta f$ method.

The time evolution for the mode amplitude of the electrostatic potential from both codes is shown in Fig.6, where the mode amplitude has grown by an order of $10^{1-2}$ for the total- $f$ code and $10^{4-5}$ for the $\delta f$ code. The measured growth rate is $\gamma=0.44 \tau_{A}^{-1}$ for the $\delta f$ code, which agrees well with the Wesson's estimation. ${ }^{24}$ The linear phase lasts for a very long time in the $\delta f$ code. When the mode amplitude becomes large, the growth rate in the $\delta f$ case reduces slightly because of the nonlinear effects (for example, the $m=1$ island 
width is relatively large compared with the minor radius of $q=1$ surface); the growth rate in this stage is almost identical to the results for the total- $f$ code. However, the growth rate measured from the total- $f$ code contains the nonlinear effecta and that is why it is slightly less than the true linear growth rate given by the $\delta f$ code. The maximum mode amplitudes are almost identical for both cases. The comparisons presented here serve to demonstrate the usefulness of the $\delta f$ code.

Above simulations are carried out by including only $n=0$ and $n= \pm 1$ modes. To verify the validity of neglecting higher $n$ modes, we have executed the simulation with the $\delta f$ method including $n=0, n= \pm 1$, and $n= \pm 2$ modes. We have chosen $\Delta t=0.87 \omega_{c i}^{-1}$ which is half of the value for the case of $n=0, \pm 1$. The time dependence of various energies is shown in Fig.12(a), which is similar to that of Fig.7 where only $n=0$ and $n= \pm 1$ modes are included. In the full reconnection phase the results for both cases are almost identical although the maximum $\mathcal{E}_{E}$ is slightly higher when $n=0, \pm 1, \pm 2$ modes are included. The growth rate $\gamma=0.43 \tau_{A}^{-1}$ for the case of $n=0, \pm 1, \pm 2$ is essentially the same as $\gamma=0.44 \tau_{A}^{-1}$ for the case of $n=0, \pm 1$. Fig.12(b) shows the components of $\mathcal{E}_{E}$ for $n=0, \pm 1, \pm 2$, respectively. We can see $n= \pm 1$ are dominant; however, we can see a small contribution of $n= \pm 2$ modes. The energy going to $n=0$ mode is negligible. The corresponding time evolution of the magnetic field structure, electrostatic potential profile and $\delta J_{z}$ (not presented in the paper) are similar to the previous case in the full reconnecton phase. Hence we can conclude that the inclusion of only $n=0, \pm 1$ modes is suffecient to simulate the full reconnection phenomena. 
Finally, let us describe some of the nonlinear phenomena after the full reconnection when the modes of $n=0, \pm 1, \pm 2$ are included. Fig. 13 shows the time evolution of the magnetic field structure in the post full reconnection phase, whereas Fig.14 shows the electrostatic potential profile. The Roman numerals in Figs.13 and 14 correspond to the different stages of the development shown in Fig.12. It is clear that the potential profile created in the full reconnection phase survives for a longer time. Due to the $E \times B$ motion driven by this potential, the plasma in the peripheral region at the opposite side of the original $x$-point comes into the core region. The magnetic reconnection again forms the new core plasma. The central $q$ value now becomes 0.95 , which is below unity but is higher than the initial value of 0.85 . These results are similar to the results of Biskamp and Drake. ${ }^{27}$ In the case only including $n=0, \pm 1$, we also observed the newly formed $q_{0}=0.95$ region. However, after this time, the two cases start to differ from each other because the $m= \pm 2$ modes are generated which demand the inclusion of $n= \pm 2$ modes because $q \simeq 1$. We have also done the simulation including up to $n= \pm 3$ modes. It is found that energy going to $n= \pm 3$ modes is quite small but the mode structure seems to be sensitive to the inclusion of $n= \pm 3$ modes after the formation of the core region of $q<1$. Hence, if we plan to investigate the phenomena long after the full reconnection phase, it may be necessary to include several additional $n$ modes. 


\section{Conclusions and Discussion}

The internal disruption of a tokamak is simulated by a three-dimensional magnetoinductive gyrokinetic particle code. In addition to a standard gyrokinetic code (total- $f$ code), a code using the nonlinear characteristic method ( $\delta f$ code $)^{15}$ is developed and is applied to the problem, where a straight tokamak model with a square cross section surrounded by a conducting wall is employed. In the toroidal direction, the periodic boundary conditon is used in the model. The linear mode structure of an unstable $m=1$, $n=1$ kinetic internal kink mode has been clearly observed especially in the $\delta f$ code because of its low noise nature. The width of the current layer around the $x$-point, where magnetic reconnection occurs, is found to be close to $c / \omega_{p e}$. This is consistent with the Wesson's prediction ${ }^{24}$ in which electron inertia has a dominant role. The characteristic time for the full reconnection also agrees with the theory. Full reconnection is observed in the simulation. After the full reconnecton, the $q$-value around the new magnetic axis is almost unity. The nonlinear behavior of the mode is found to be quite similar for both codes. The simulation results support a two-phase model of Biskamp and Drake. ${ }^{27}$ As a result of full reconnection there remains the electrostatic potential structure inside the $\mathrm{q}=1$ surface. When the full reconnection phase is finished, $q \simeq 1$ in the newly formed core region. Hence, the electroststic mode pattern and the magnetic field structure are almost completely synchronized. As a result, there is almost no change in the electrostatic potential along the magnetic field. Therefore, the mode pattern survives for a long time. 
Since there is a plasma flow in this shearless area due to the $E \times B$ drift even after the full reconnection phase, the flow pushes the peripheral plasma outside the $q=1$ surface into the central region. A further change in topology of the magnetic field structure is observed due to this new phase of magnetic reconnection. Consequently, the central safety factor then goes down to 0.95 , which is slightly closer to the original central safety facter of 0.85 . Further simulation is being conducted to study this phenomena and the results will appear elsewhere.

Finally, let us discuss the system size used in the simulation. The choise of $c / \omega_{p e}=4$ for the skin depth to simulate a computational domain of $16 c / \omega_{p e} \times 16 c / \omega_{p e} \times 8000 c / \omega_{p e}$ is due to the fact that we must have the necessary resolution to treat the current layer physics near the $x$-point. Although realistic simulation may need a bigger simulation volume, we believe the results presented here have captured the dominant physics of the kinetic internal kink mode. The results presented here have demonstrated the effectiveness of using the gyrokinetic particle simulation to investigate the kinetic modifications on MHD modes. It also pointed out the fact that more powerful massively parallel computers are needed for more realistic simulations, in which a system with uneven grid to account for the skin depth near the $q \simeq 1$ surface may be necessary. 


\section{Acknowledgement}

The authors wish to thank Professor J.M. Dawson at University of California at Los Angeles, Professors T. Kamimura and T. Sato at National Institute for Fusion Science, Dr. S. Tokuda at Japan Atomic Energy Research Institute, and Professor O. Fukumasa at Yamaguchi University for the stimulating discussions and continuous encouragement. They also wish to thank Drs. S. Parker and J. Cummings at Princeton Plasma Physics Laboratory (PPPL) and Professor M. Wakatani at Kyoto University for their valuable discussions and critical comments. The computer system at the National Institute for Fusion Science has been used for the numerical calculations.

One of us (HN) was sponsored by the US/Japan Joint Institute of Fusion Theory exchange program for his visit to PPPL and he would like to acknowledge the hospitality of his host. The present work is also supported in part by the U. S. Department of Energy under Contracts No. DE-AC02-76-CHO3073 at PPPL. 


\section{Figures}

Fig.1 Time evolution for the perturbed quantities associated with kinetic $\left(\mathcal{E}_{K}\right)$, electric field $\left(\mathcal{E}_{E}\right)$, magnetic field $\left(\mathcal{E}_{B}\right)$, and total $\left(\mathcal{E}_{T}\right)$ energy. Each represents its deviation from the initial energy level and is normalized by the initial total energy $\mathcal{E}_{T 0}$. $\left(\mathcal{E}_{E}\right.$ is identical to the fluid kinetic energy.) Total $f$ code.

Fig.2 Time evolution for the magnetic field structure at $z=0$ cross section. The number corresponds to the time sequence in Fig.1. Total- $f$ code.

Fig.3 Time evolution for the electrostatic potential contours at $z=0$ cross section. The number corresponds to the time sequence in Fig.1. Total- $f$ code.

Fig.4 Time evolution of the perturbed current density, $\delta J_{z}=J_{z}-J_{z 0}$, at the $z=0$ cross section. The data is time-avareged with a width of $\pm 87 \omega_{c i}^{-1}$. The number corresponds to the time sequence in Fig.1. Total- $f$ code.

Fig.5 Contour plot of $\delta J_{z}$ at $z=0$ cross section (a) and its profile along the cut $l$ (b) at time sequence IV in Fig.1. The cut crosses the center of the box and the $x$-point in (a) and the collisionless electron skin depth is shown in (b). Total- $f$ code.

Fig.6 Time dependence for the amplitude of the electrostatic potential taken at $z=0$ cross section from the total- $f$ and $\delta f$ codes.

Fig.7 Time dependence for the perturbed quantites associated with kinetic $\left(\mathcal{E}_{K}\right)$, electric field $\left(\mathcal{E}_{E}\right)$, magnetic field $\left(\mathcal{E}_{B}\right)$, and total $\left(\mathcal{E}_{T}\right)$ energy. Each represents its deviation 
from its initial energy level and is normalized by initial total energy $\mathcal{E}_{T 0}$. ( $\mathcal{E}_{E}$ is identical to the fluid kinetic energy.) $\delta f$ code.

Fig.8 Time evolution for the magnetic field structure at $z=0$ cross section. The number corresponds to the time sequence in Fig.7. $\delta f$ code.

Fig.9 Time evolution of the electrostatic potential contour at $z=0$ cross section. The number corresponds to the time sequence in Fig.7. $\delta f$ code.

Fig.10 Snap shots for the time evolution of the perturbed current density, $\delta J_{z}$, at $z=0$ cross section. The number corresponds the time sequence in Fig.7. $\delta f$ code.

Fig.11 Contour plot of $\delta J_{z}$ at $z=0(\mathrm{a})$ and its profile along the cut $l(\mathrm{~b})$ at time sequence I in Fig.7. The cut crosses the center of the box and the $x$-point as shown in (a) and the collisionless electron skin depth is shown in (b). $\delta f$ code.

Fig.12 Time evolution for the perturbed quantities associated with (a) $\mathcal{E}_{K}, \mathcal{E}_{E}, \mathcal{E}_{B}, \mathcal{E}_{T}$ and (b) $n=0, \pm 1, \pm 2$ components of $\mathcal{E}_{E}$. Each represents its deviation from the initial energy level and is normalized by the initial total energy $\mathcal{E}_{T 0} . \delta f$ code with $n=0, \pm 1, \pm 2$ modes.

Fig.13 The magnetic field structure at $z=0$ after the full reconnection phase. $\delta f$ code with $n=0, \pm 1, \pm 2$ modes. The number corresponds to the time sequence in Fig.12.

Fig.14 Contour plots of the potential at $z=0$ after the full neconnectin phase. $\delta f$ code with $n=0, \pm 1, \pm 2$ modes. The number corresponds to the time sequence in Fig.12. 


\section{References}

${ }^{1}$ W. W. Lee, J. Comp. Phys. 72, 243 (1987).

${ }^{2}$ T. S. Hahm, W. W. Lee, and A. Brizard, Phys. Fluids, 31, 1940 (1988).

${ }^{3}$ T. S. Hahm, Phys. Fluids, 31, 2670 (1988).

${ }^{4}$ A. J. Brizard, J. Plasma Phys. 41, 541 (1989).

${ }^{5}$ C. K. Birdsall and A. B. Langdon, "Plasma Physics via Computer Simulation", McGraw-Hill, New York (1985).

${ }^{6}$ J. M. Dawson, V. Decyk, and R. Sydora, Physics Today, 47, 64 (1993).

${ }^{7}$ D. C. Barnes et al., "Computing at the leading edge", National Energy Research Supercomputer Center, Lawrence Livermore National Laboratory Report UCRL-TB$111084,5(1993)$.

${ }^{8} \mathrm{H}$. Naitou, Journal of Plasma and Fusion Science 70, 135 (1994)(in Japanese).

${ }^{9}$ R. D. Sydora, Phys. Fluids B, 2, 1455 (1990).

${ }^{10}$ S. E. Parker, W. W. Lee, R. A. Santoro, Phys. Fluids B, 71, 2042 (1993).

${ }^{11}$ J. A. Krommes, W. W. Lee and C. Oberman, Phys. Fluids, 29, 2421 (1986).

${ }^{12}$ A. M. Dimits, Ph.D. Thesis, Princeton University (1988). 
${ }^{13}$ M. Kotschenruether, Bull. Am. Phys. Soc. 34, 2107 (1988).

${ }^{14}$ A. M. Dimits and W. W. Lee, J. Comp. Phys.107, 309 (1993).

${ }^{15}$ S. E. Parker and W. W. Lee, Phys. Fluids B, 5, 77 (1993).

${ }^{16}$ K. McGuire et. al., Phys. Rev. Letters 50, 891 (1983).

${ }^{17}$ C.Z. Cheng, M.S. Chance, Phys. Fluids 29, 3695 (1986).

${ }^{18}$ D. J. Campbell et al., Phys. Rev. Lett. 60, 2148 (1988).

${ }^{19}$ A. W. Edwards et al., Phys. Rev. Letters 57, 210 (1986).

${ }^{20}$ H. Soltwisch, Rev. Sci. Instrum. 59, 1599 (1988).

${ }^{21}$ R. C. Wolf et al., Nuclear Fusion 33, 663 (1993).

${ }^{22}$ M. Yamada, F. Levinton, N. Pomphrey, R. Budney, J. Manickam, and Y. Nagayama, Princeton Plasma Physics Laboratory Report PPPL-3004 (1994).

${ }^{23}$ B. B. Kadomtsev, Fiz. Plazmy 1, 710 (1975) [Sov. J. Plasma Phys. 1, 389 (1976)].

${ }^{24}$ J. A. Wesson, Nuclear Fusion 30, 2545 (1990).

${ }^{25}$ V.M. Vasyliunas, Rev. Geophys. and Space Phys. 13, 303 (1975).

${ }^{26}$ Ya. I. Kolesnichenco, Yu. V. Yakovenko, D Anderson, M. Lisak, and F. Wising, Phys. Rev. Lett.,68, 3881 (1992). 
${ }^{27}$ D. Biskamp and J.F. Drake, Phys. Rev. Lett., 73, 971 (1994).

${ }^{28}$ H. R. Strauss, Phys. Fluids, 19, 134 (1976).

${ }^{29}$ J. M. Hammersley and D. C. Handscomb, in "Monte Carlo Methods", Methuen Co Ltd, London, 133 (1964). 


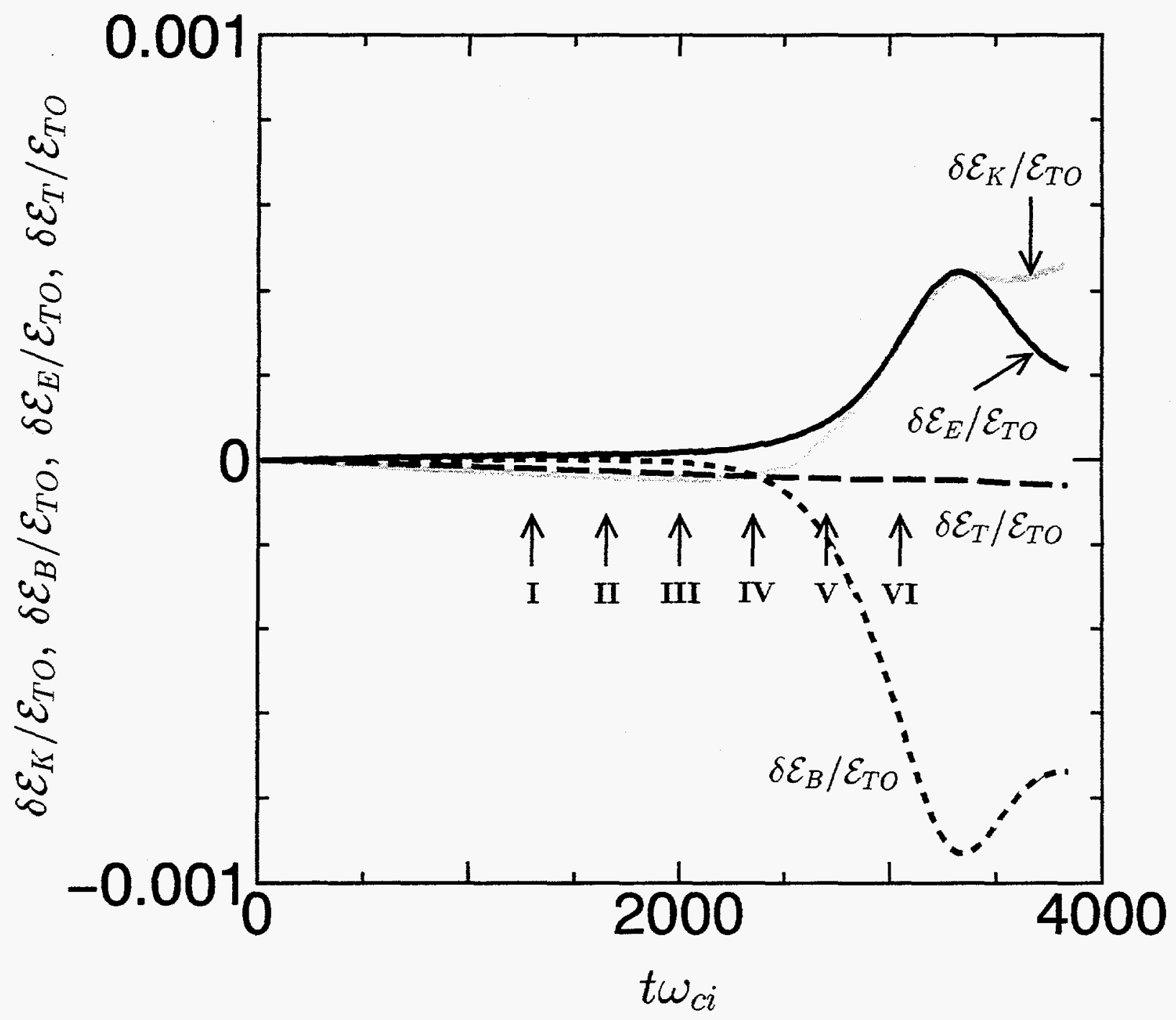

Fig. 1 

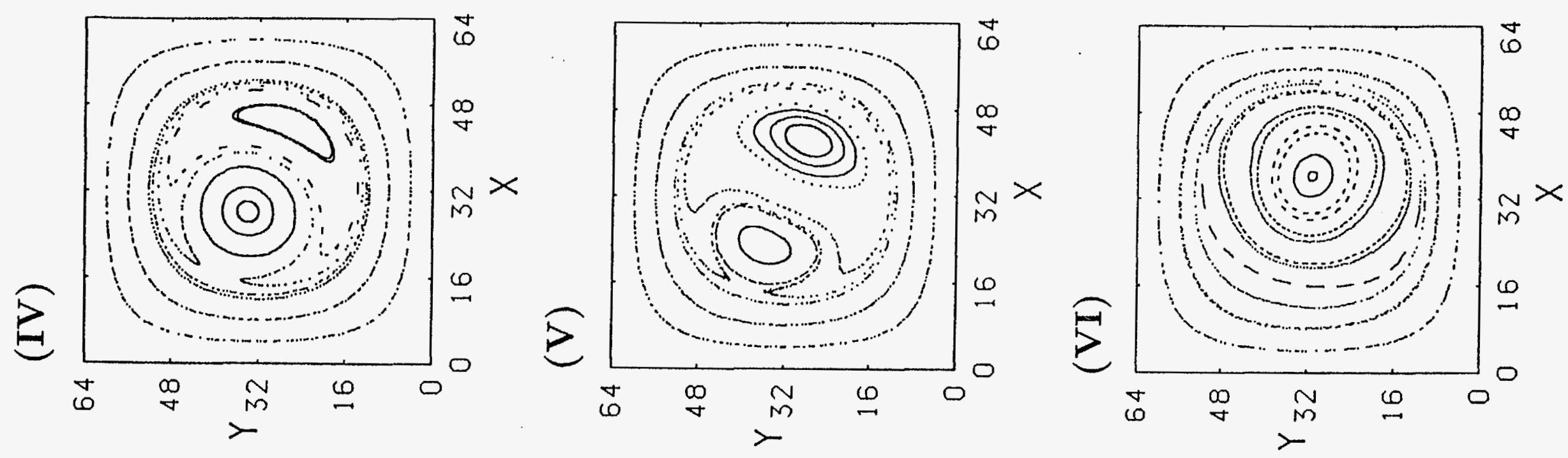

$\underset{\substack{N \\ i=1 \\ i=1}}{m}$
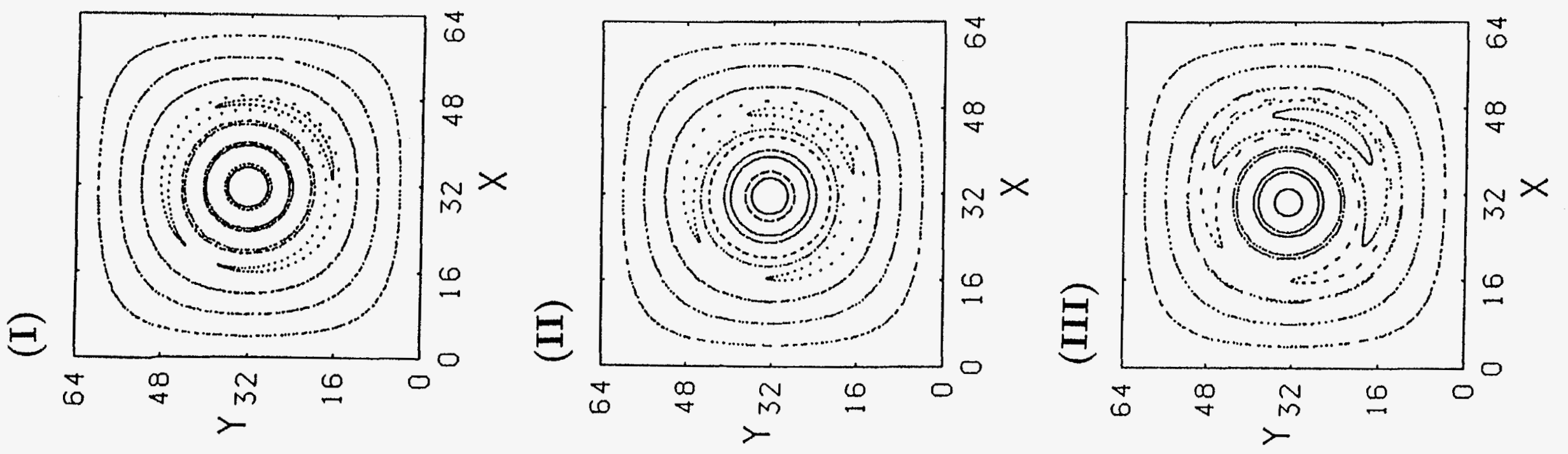
(I)

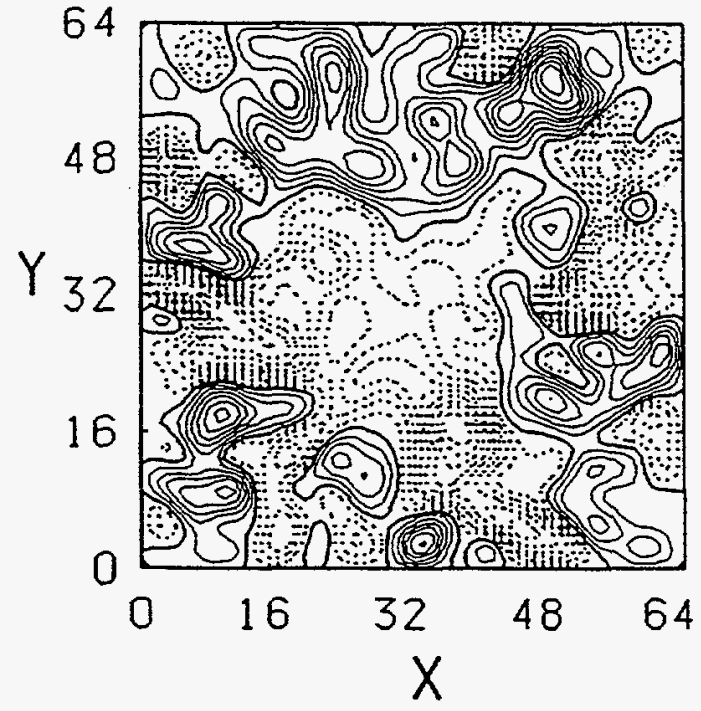

(II)

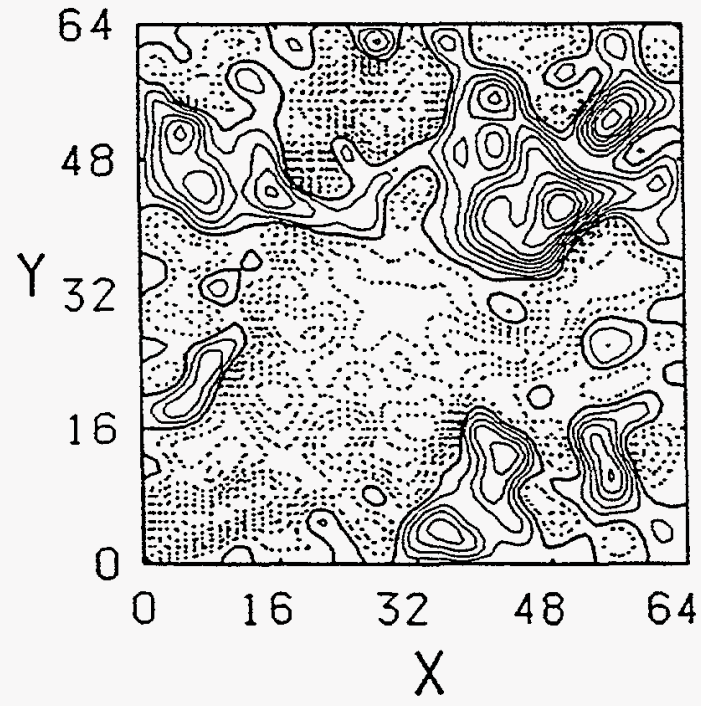

(III)

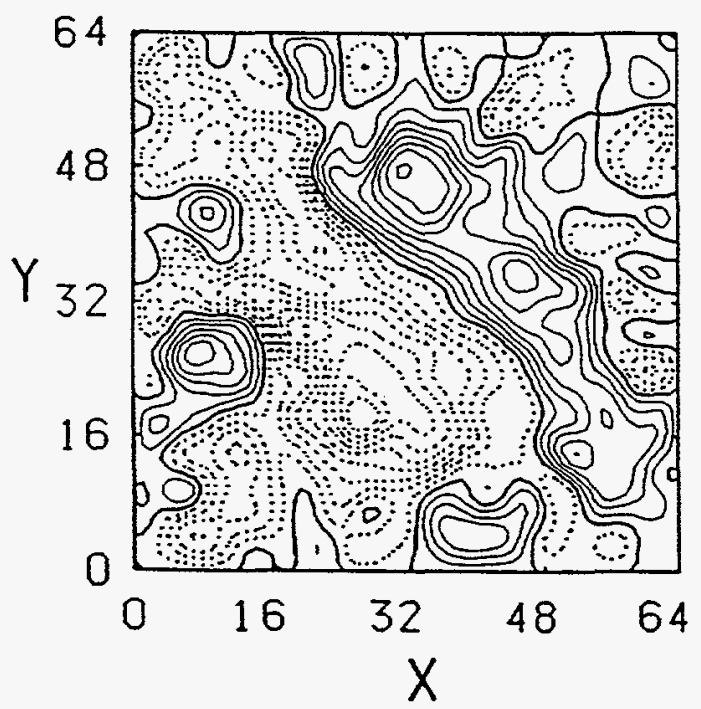

(IV)

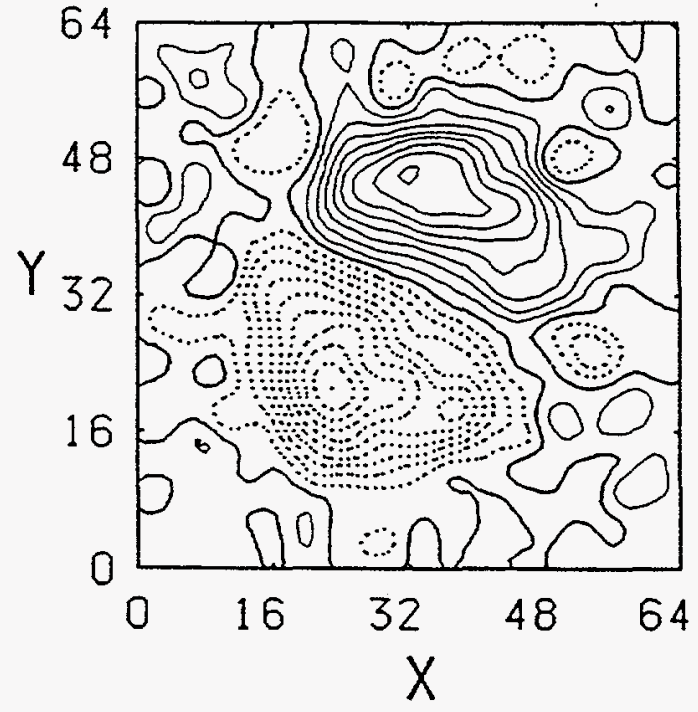

(V)

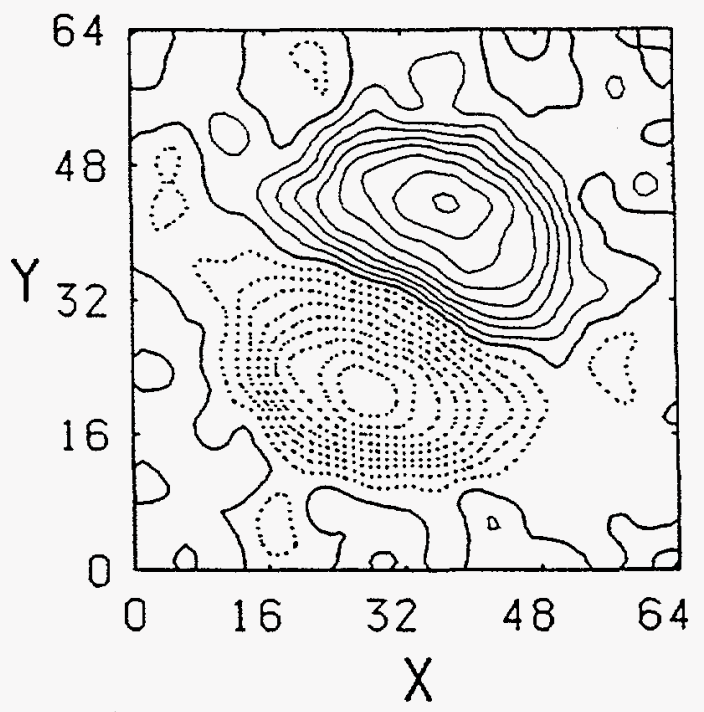

(VI)

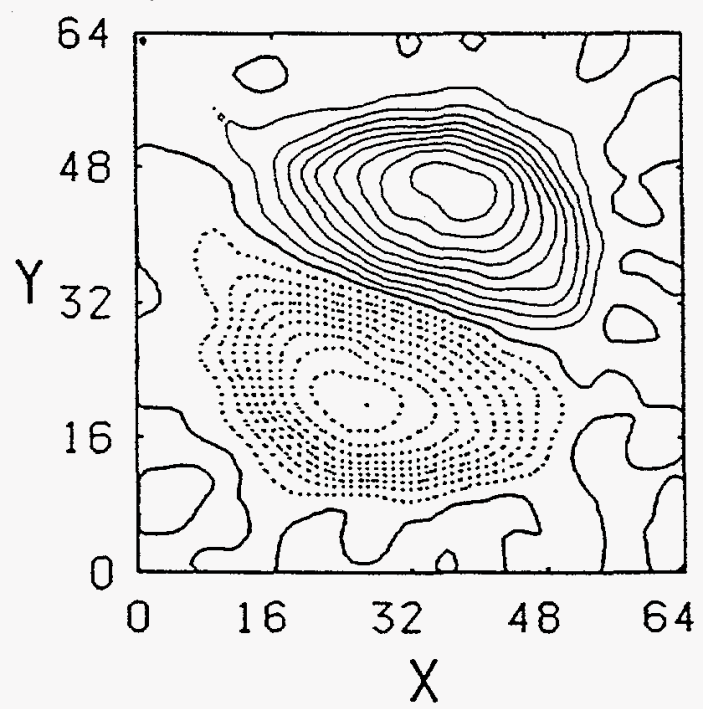

Fig. 3 
(I)

8.1E-03

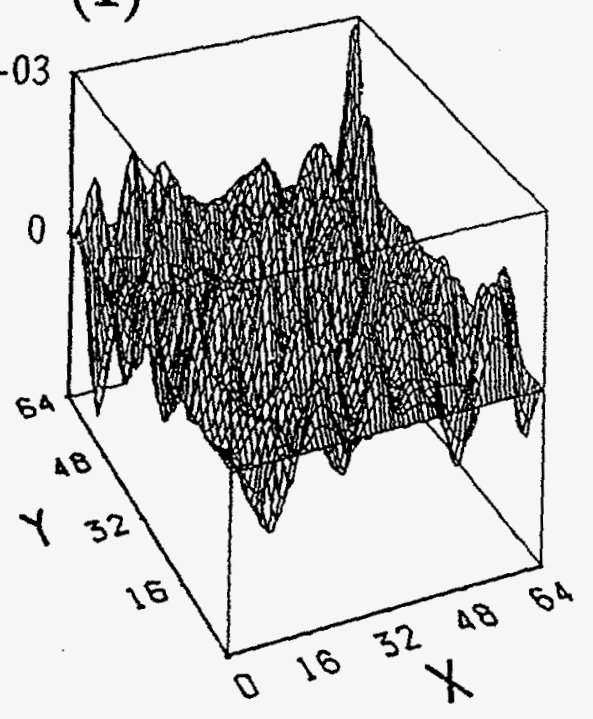

(II)

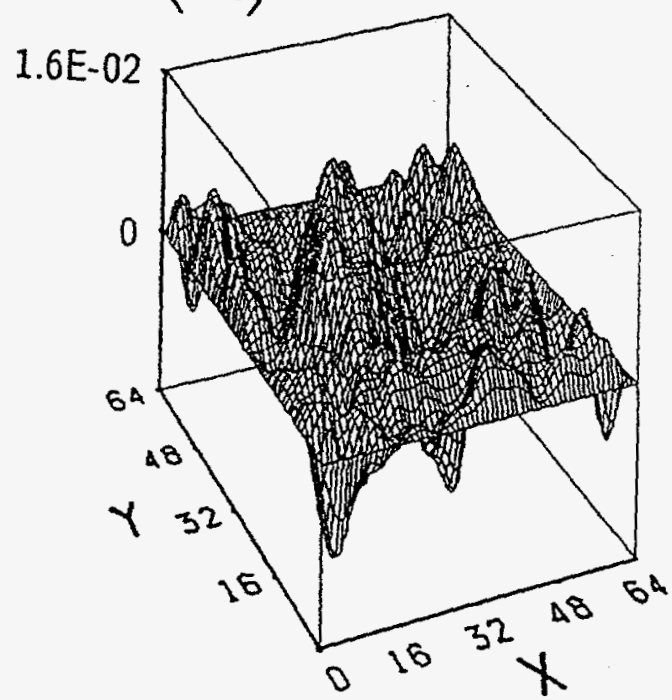

(III)

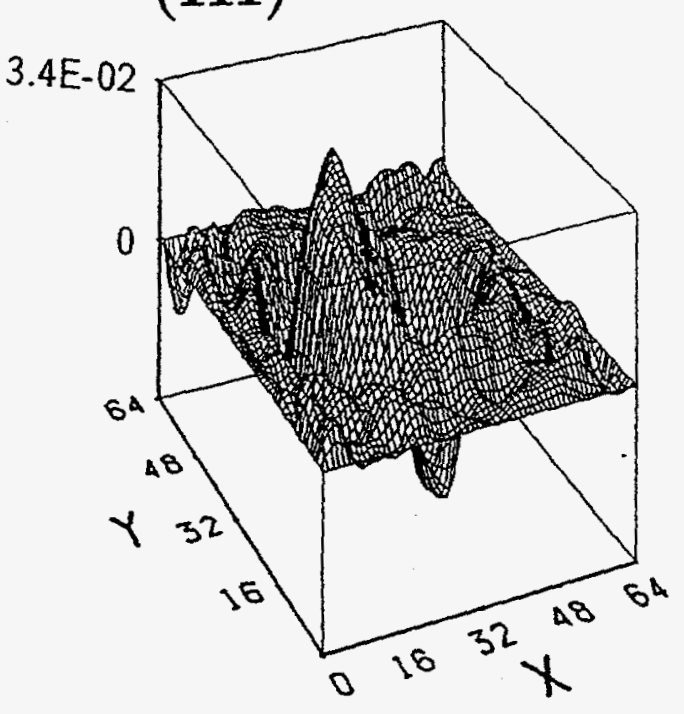

(IV)

8.5E-02

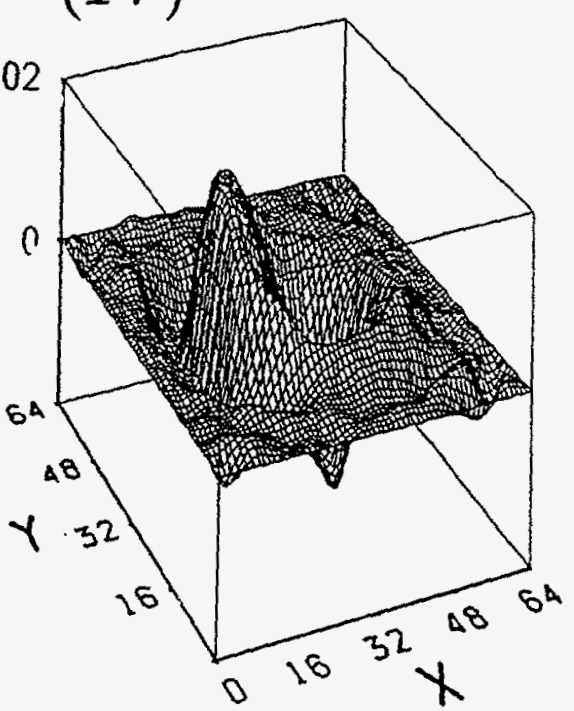

(V)

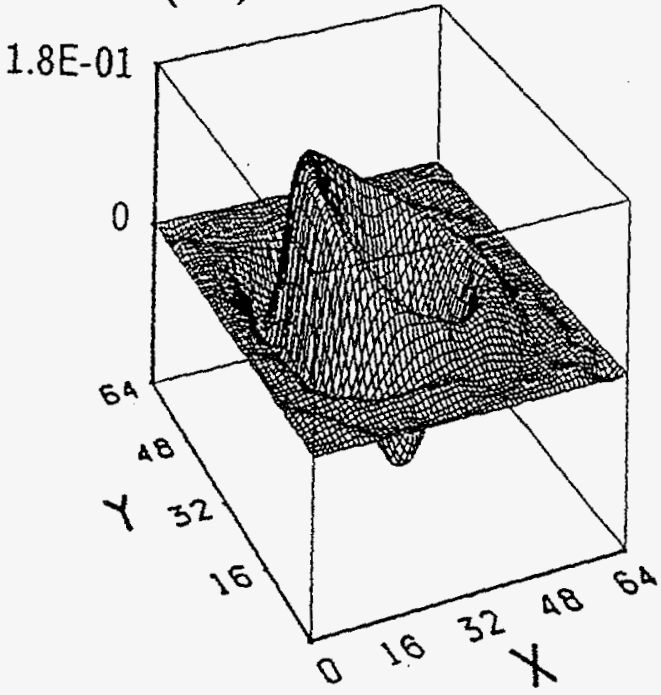

(VI)

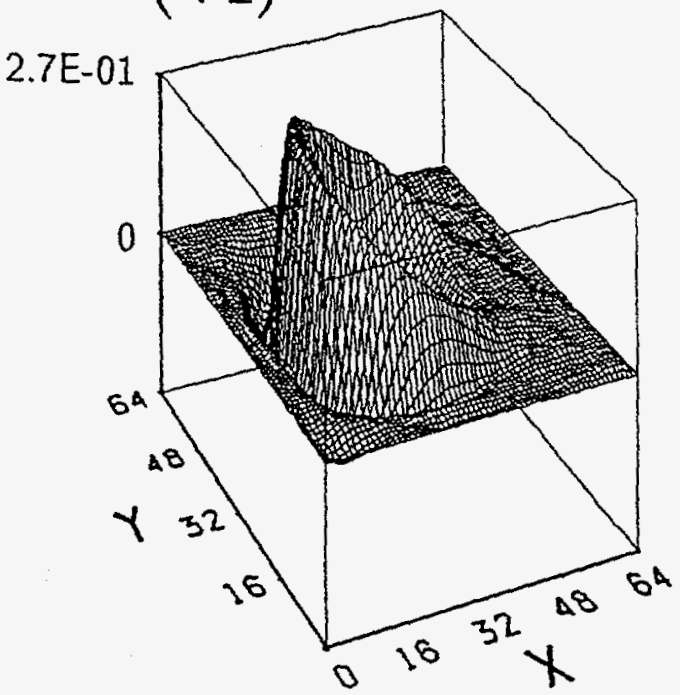

Fig. 4 
(a)

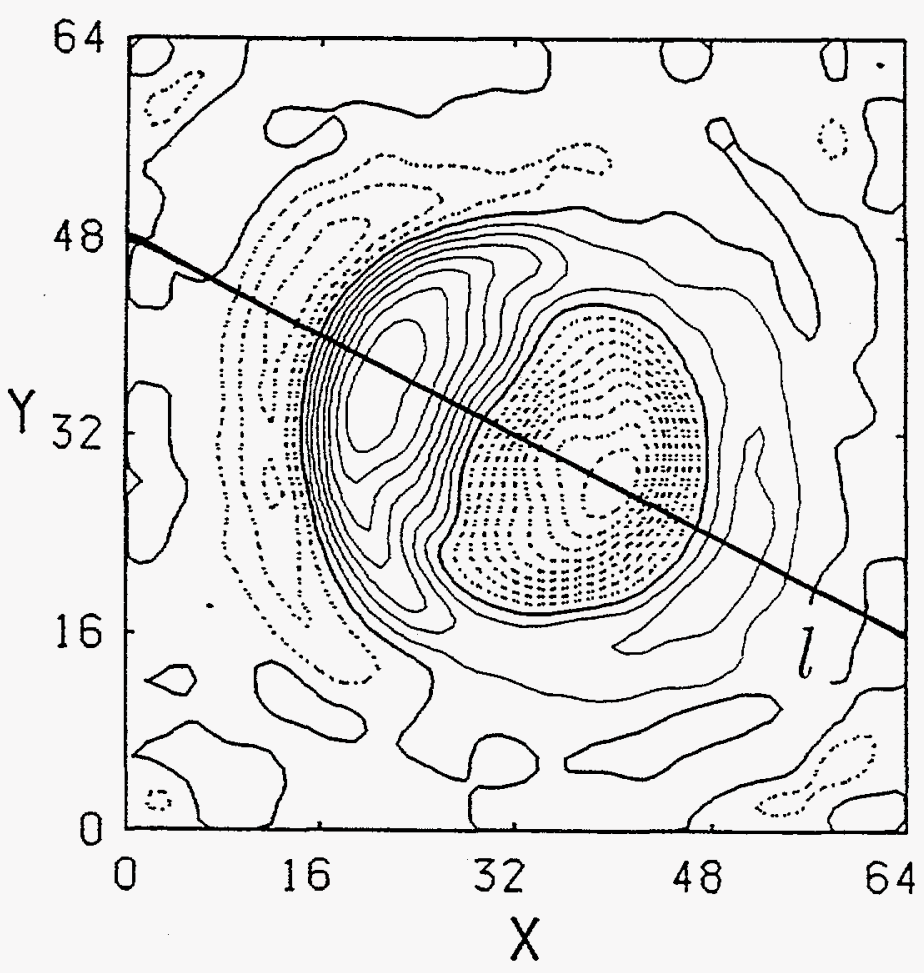

(b)

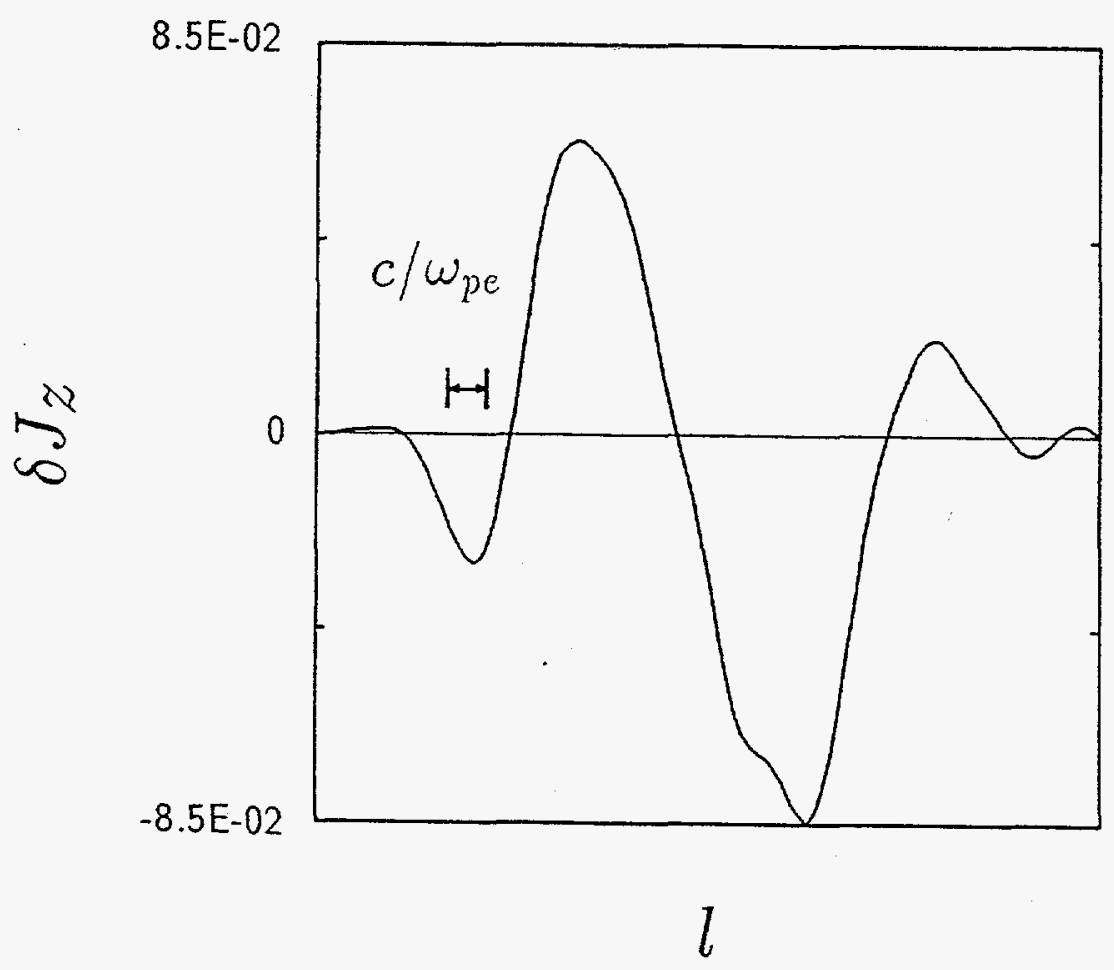

Fig. 5 


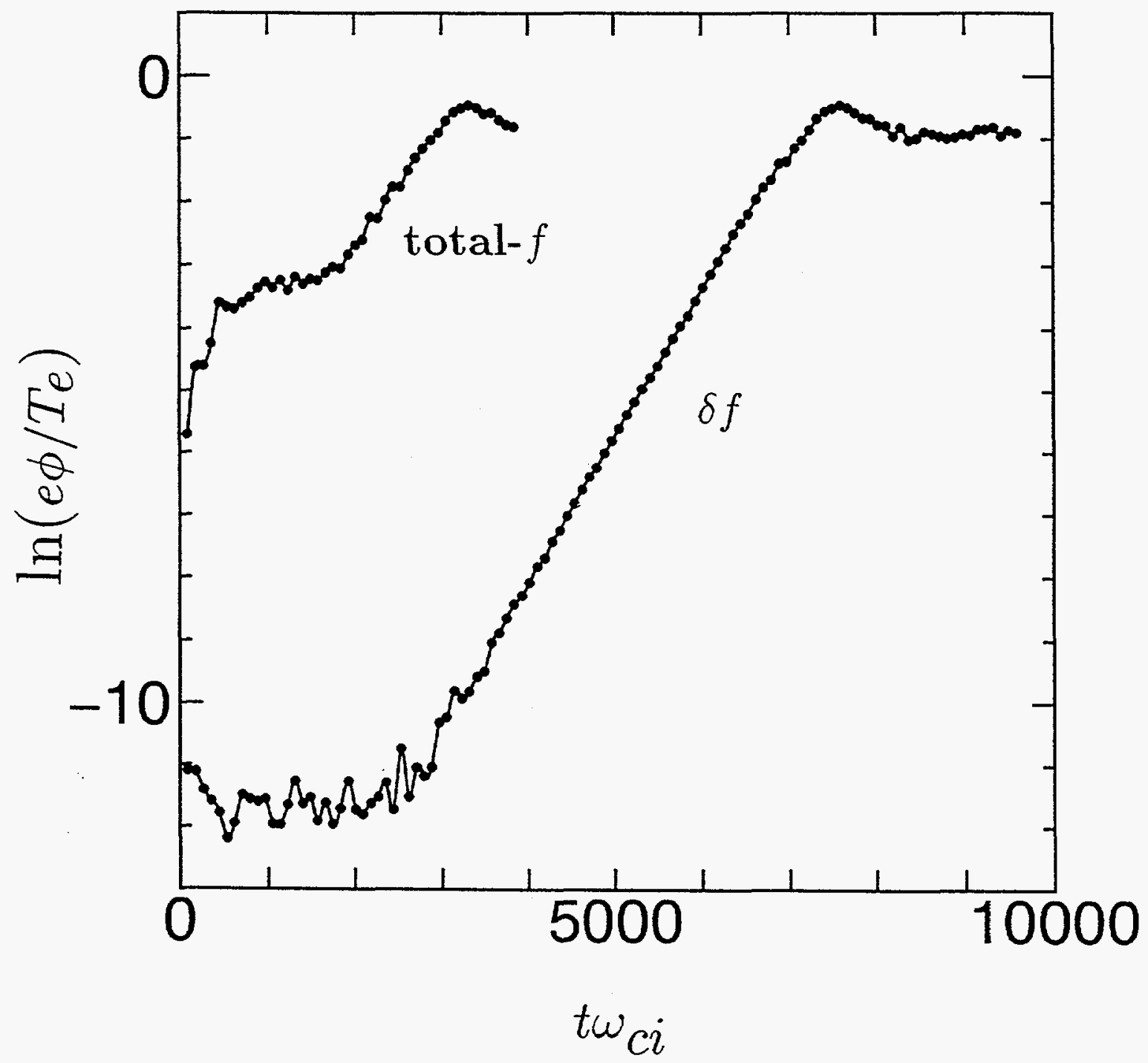

Fig. 6 


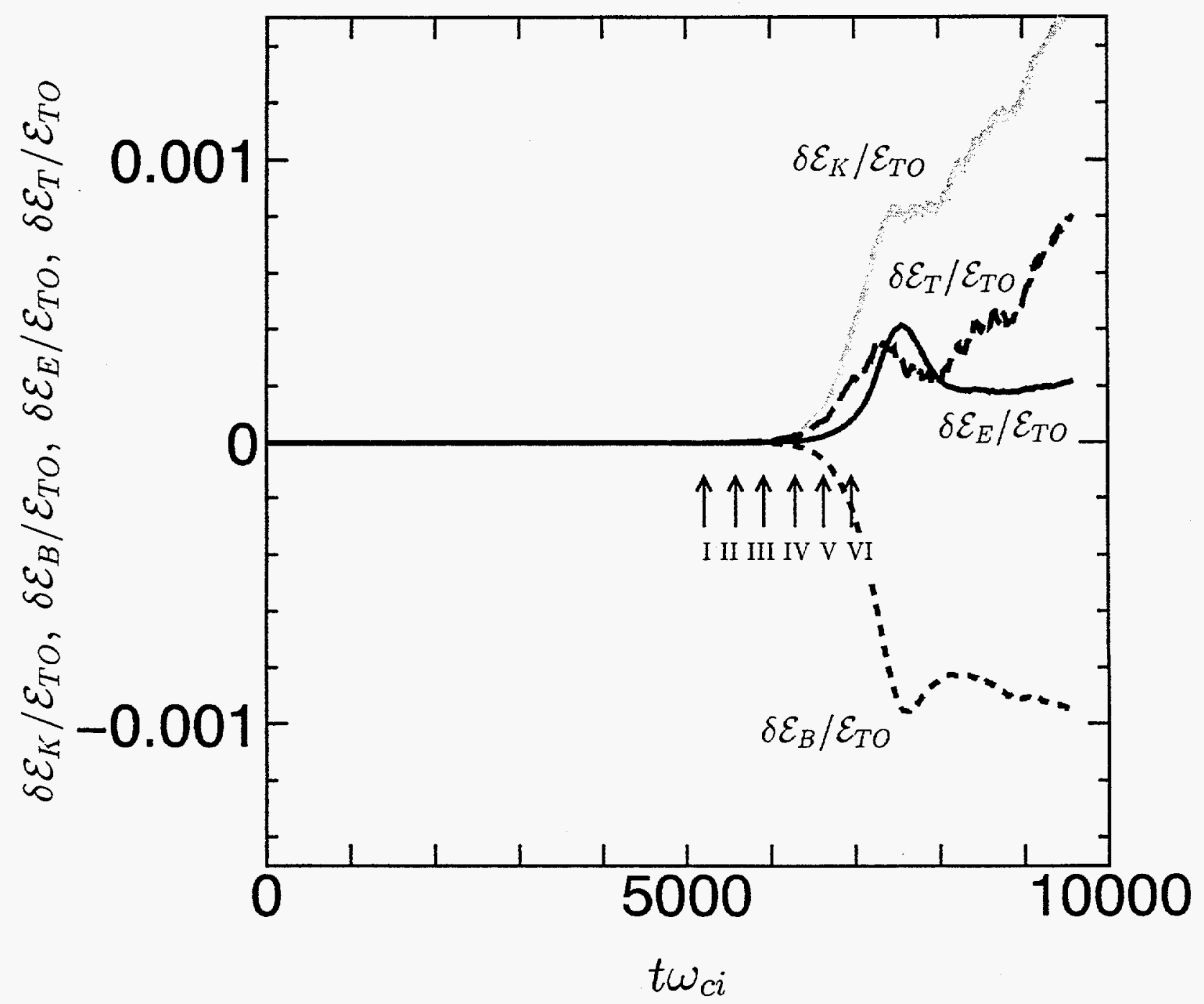

Fig. 7 
$X$

$\forall 9 \quad 87 \quad$ ZE $9 I \quad 0$

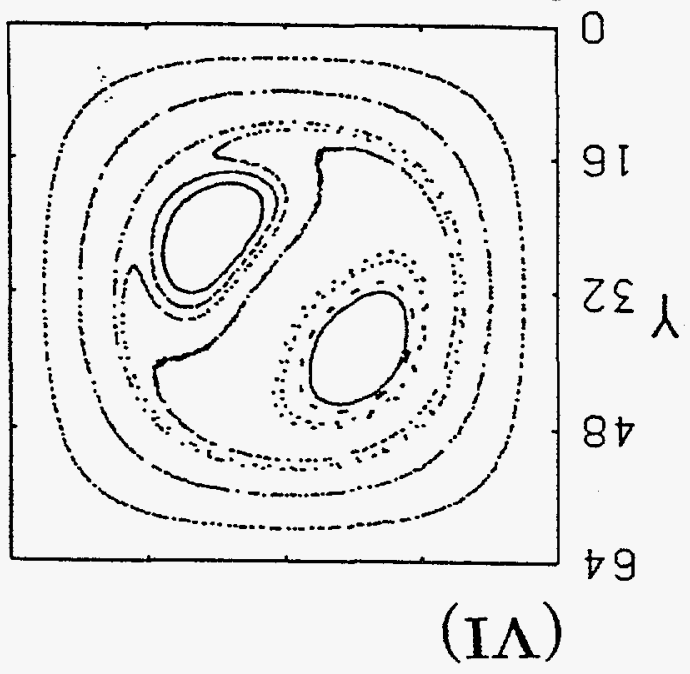

$X$

$\nabla 9$ 8t $2 E$ 9I 0

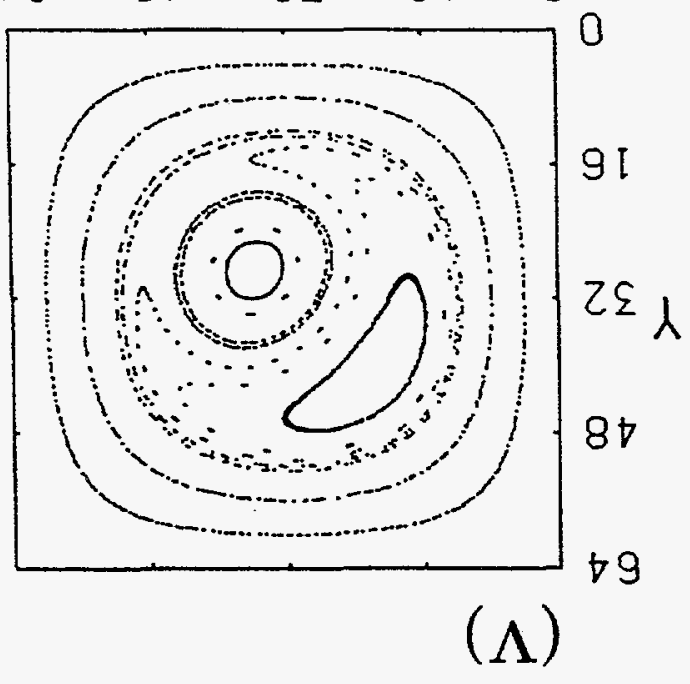

$X$

$\begin{array}{lllll}7 & 8 \nabla & 2 \varepsilon & 91 & 0\end{array}$

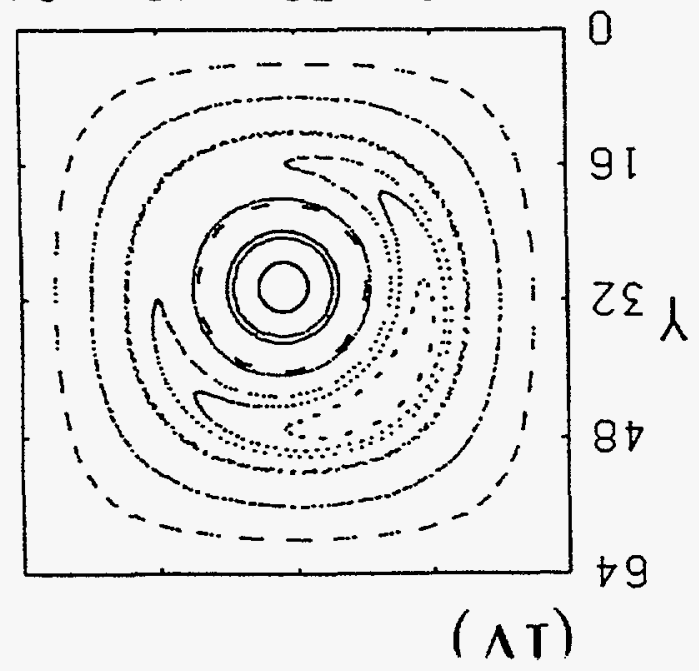

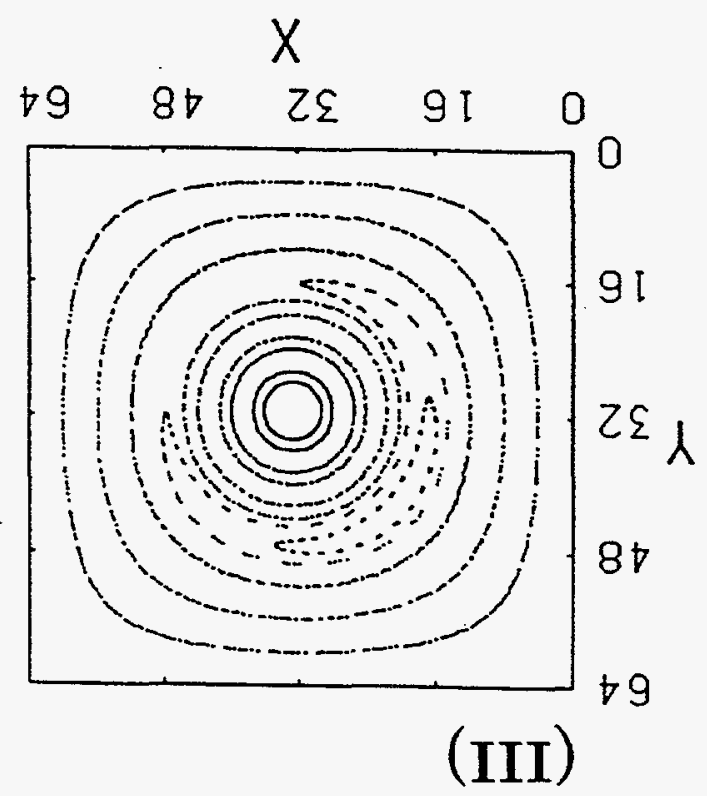

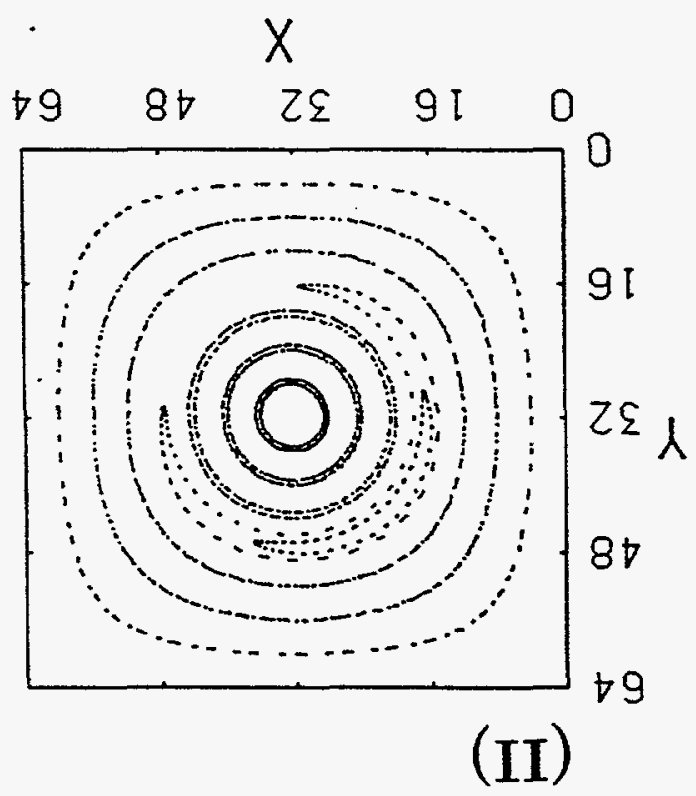

$x$

$\begin{array}{llllll}9 & 87 & 2 \varepsilon & 91 & 0\end{array}$

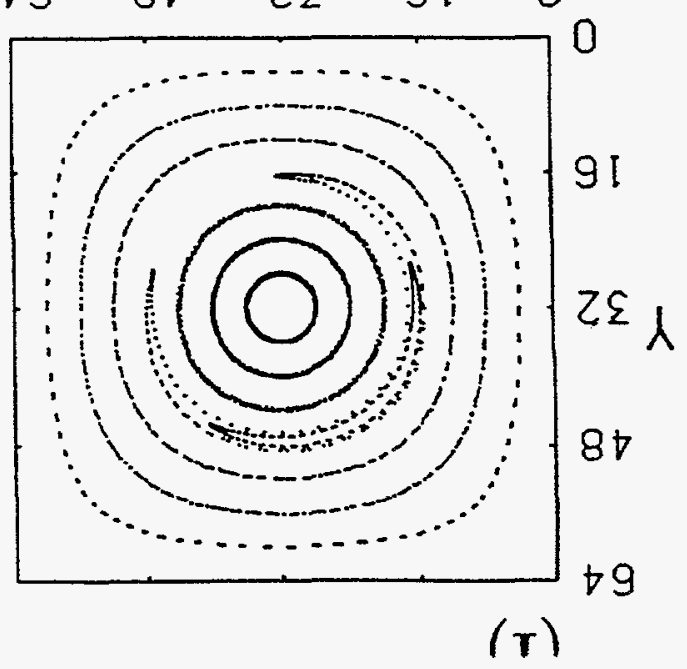


(I)

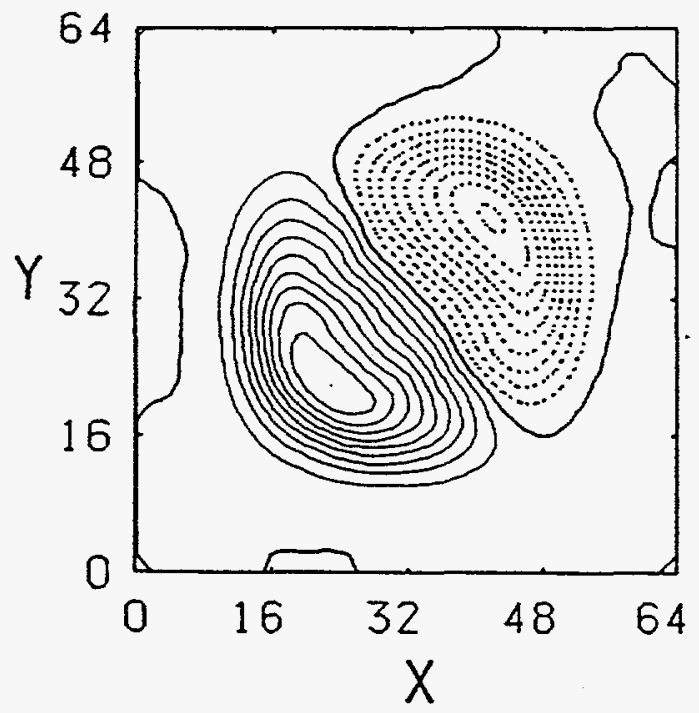

(II)

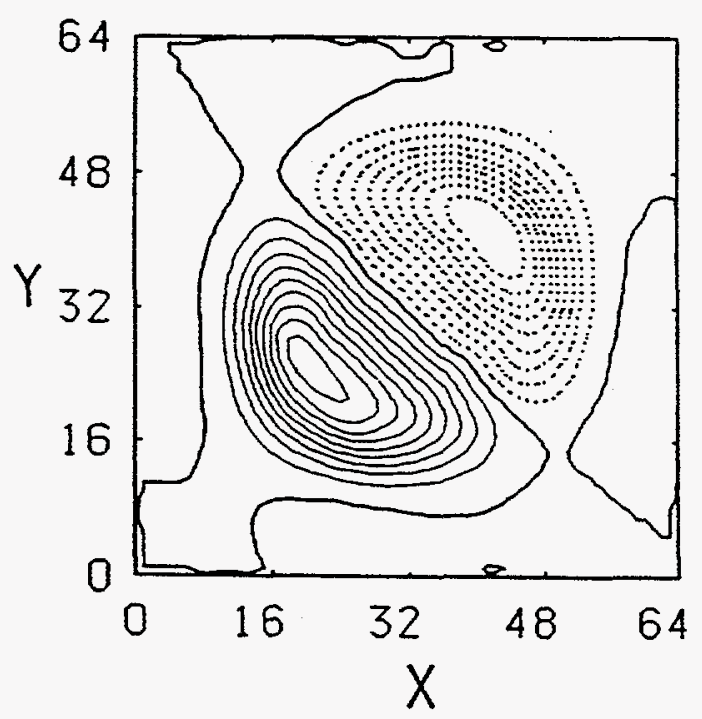

(III)

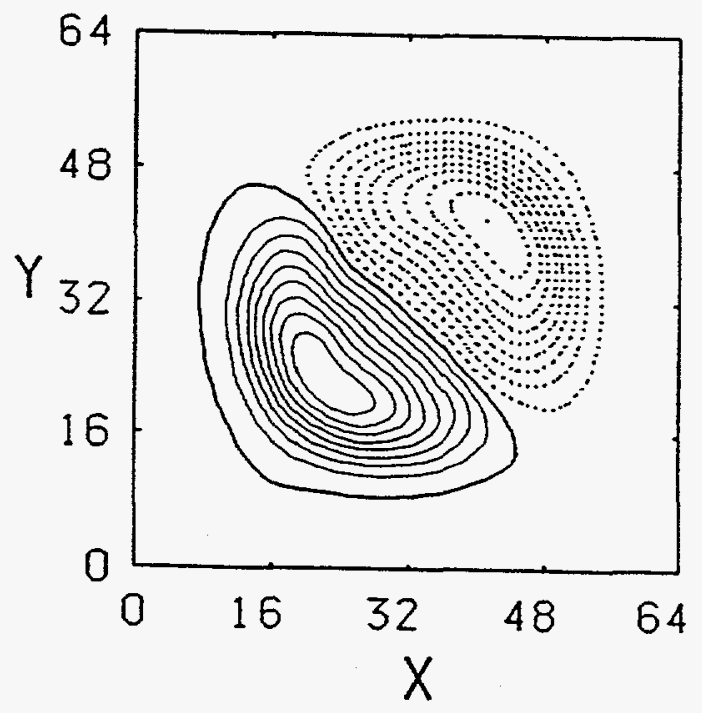

(IV)

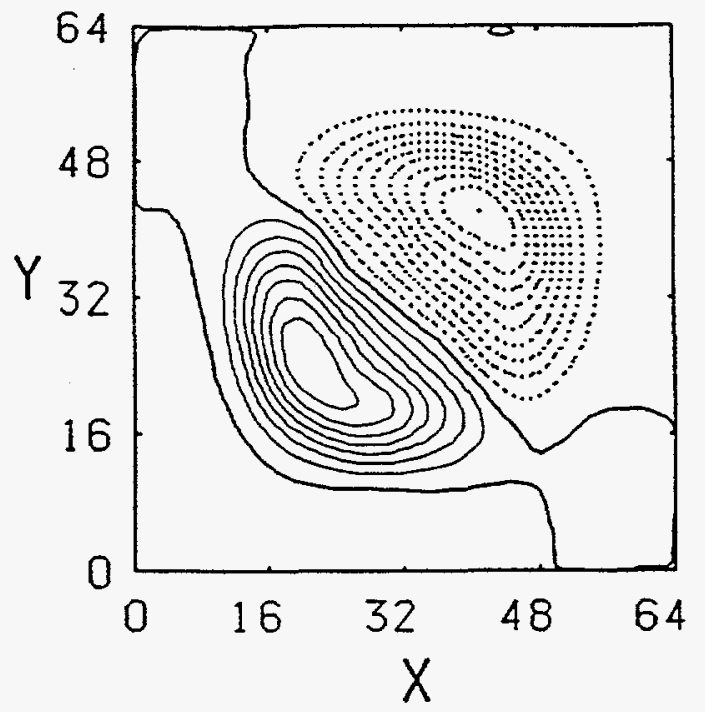

(V)

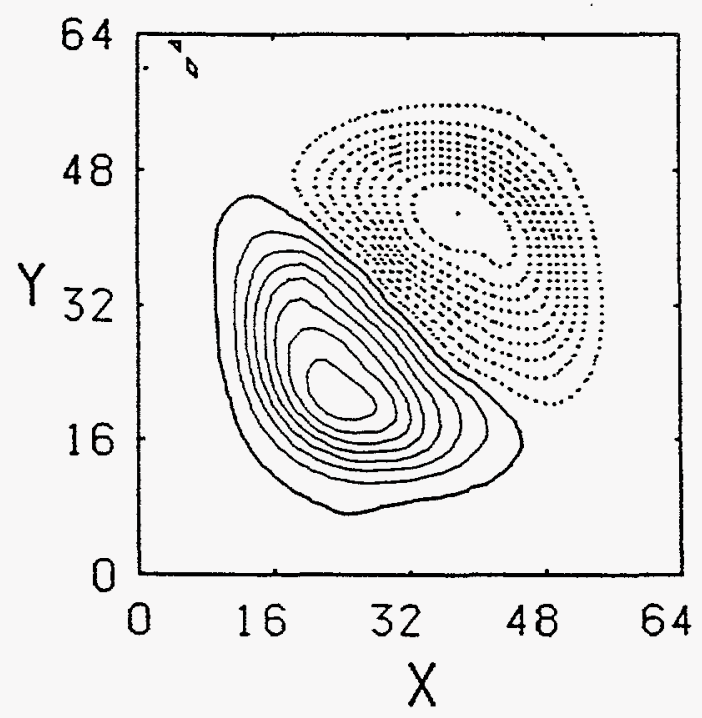

(VI)

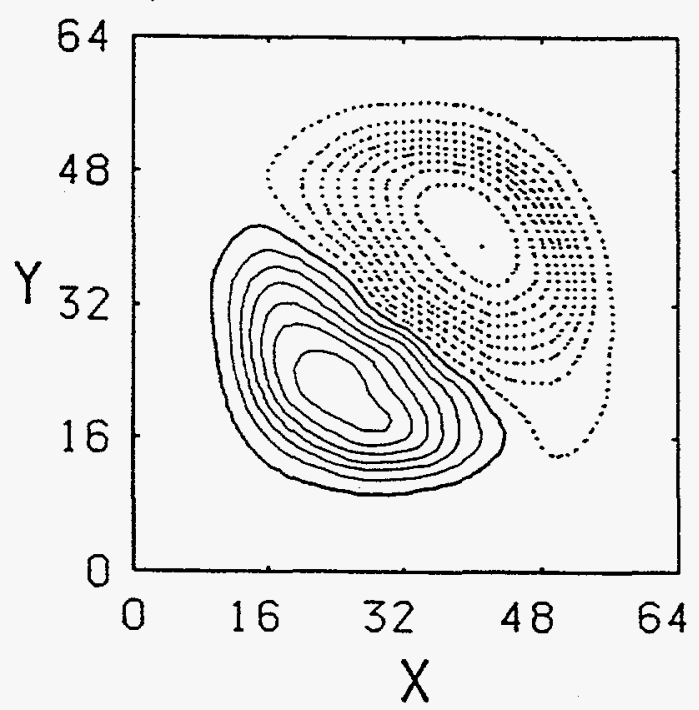

Fig. 9 
(I)

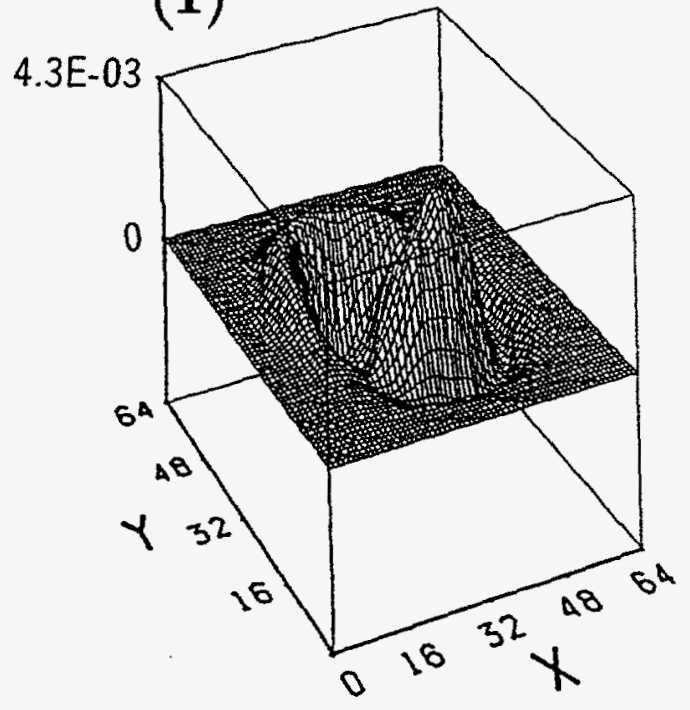

(II)

8.6E-0

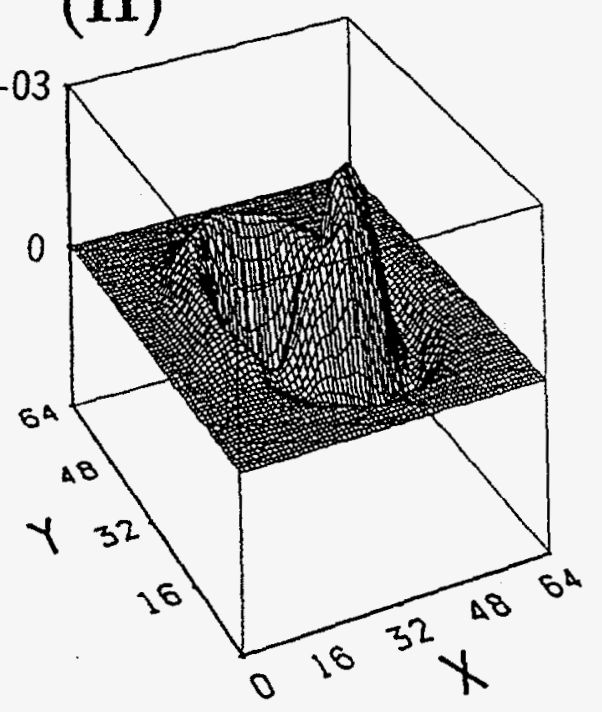

(III)

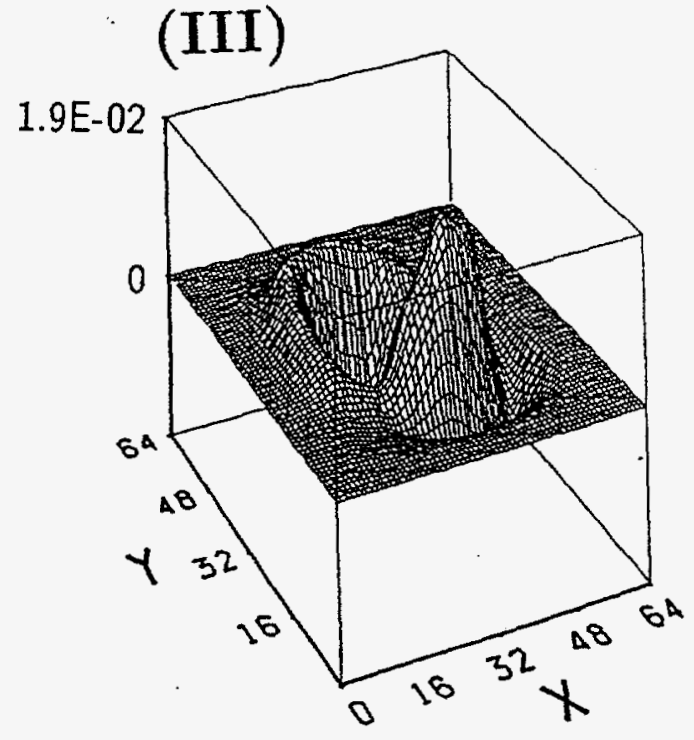

(IV)

4.3E-02

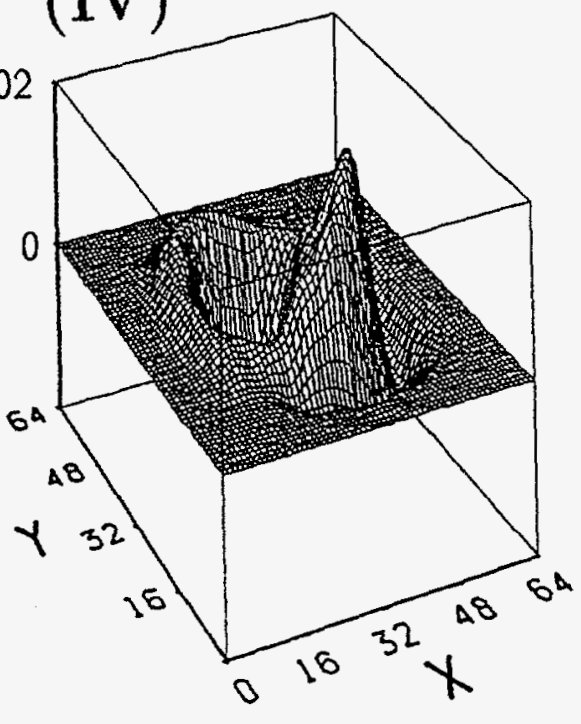

(V)

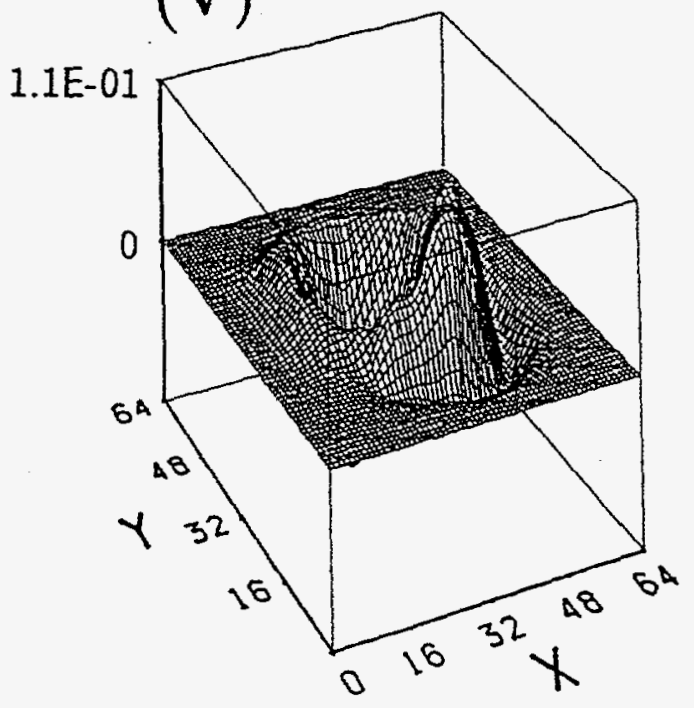

(VI)

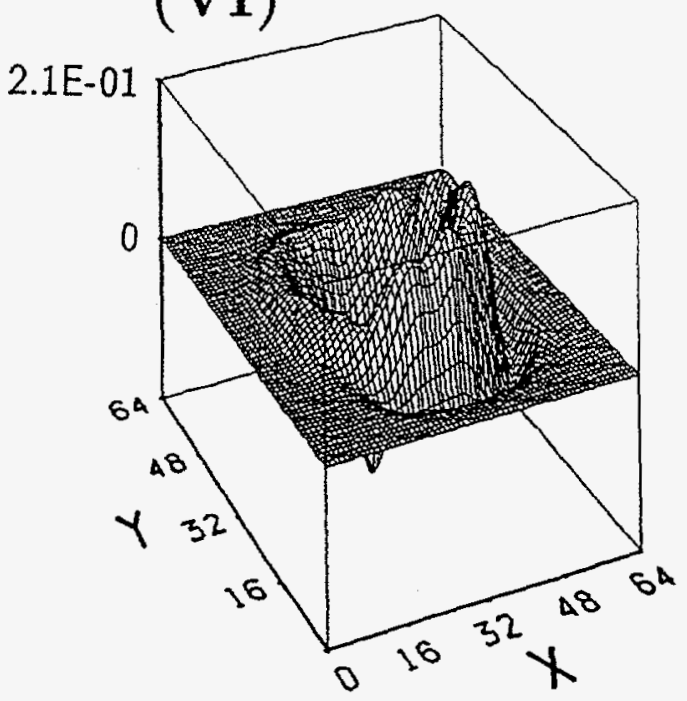

Fig. 10 
(a)

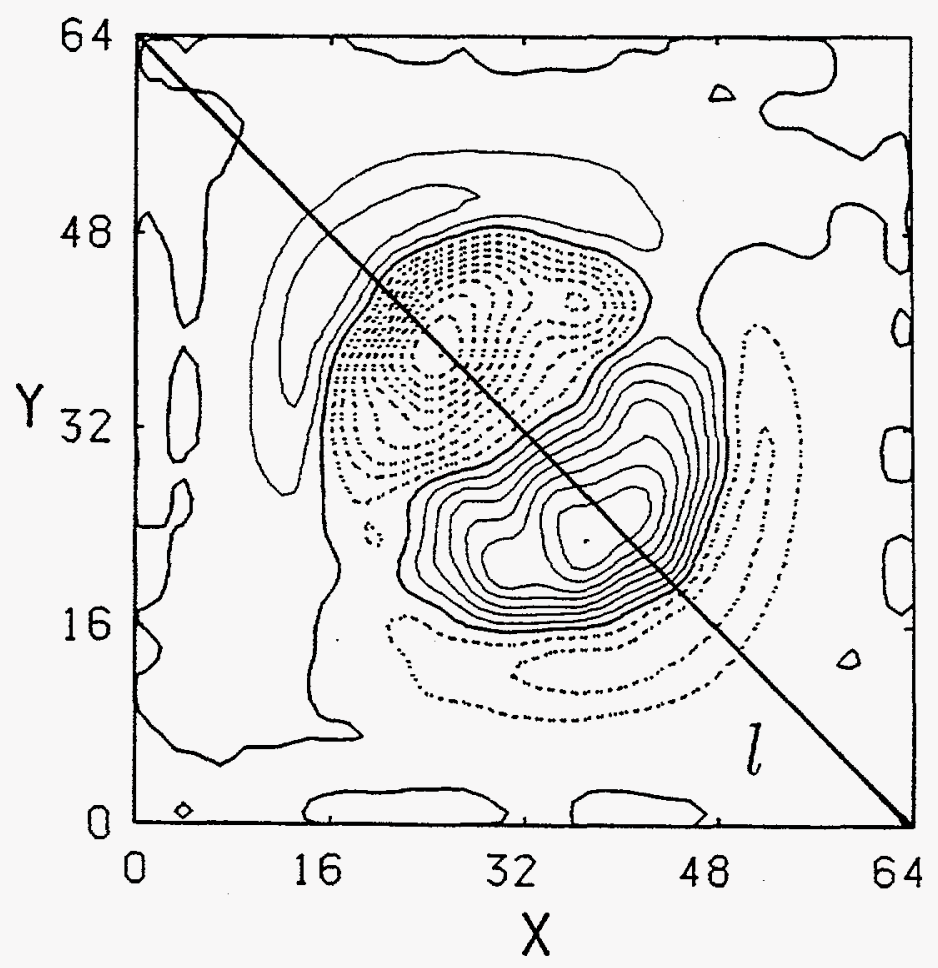

(b)

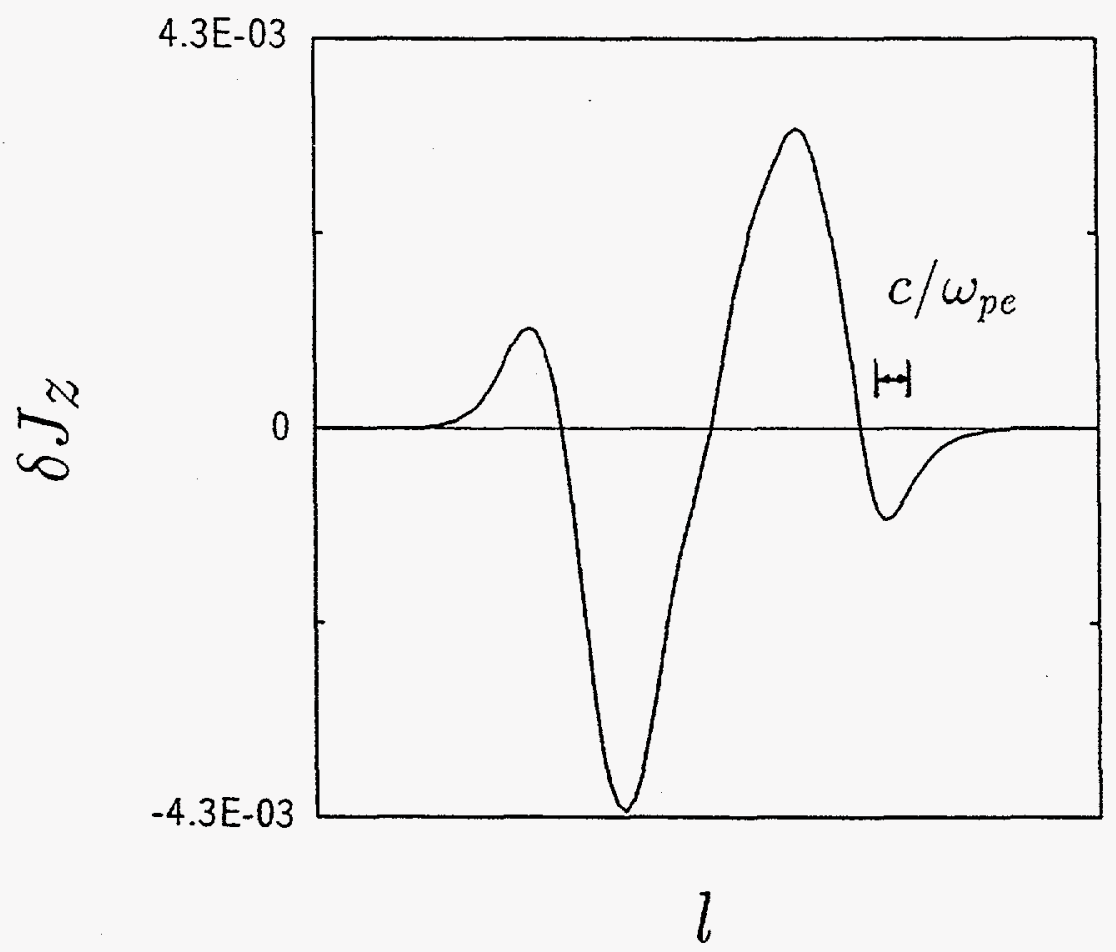

Fig. 11 
(a)

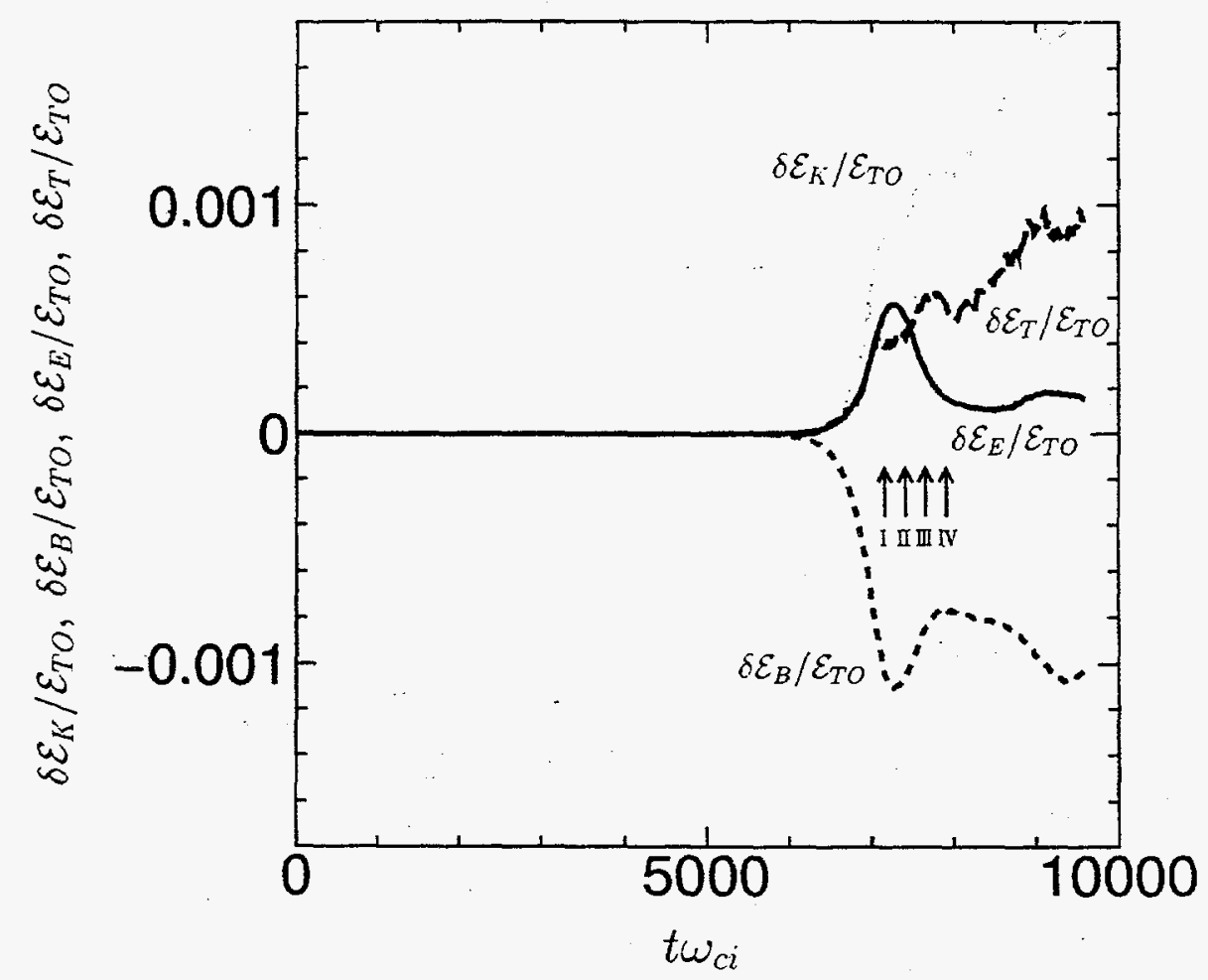

(b)

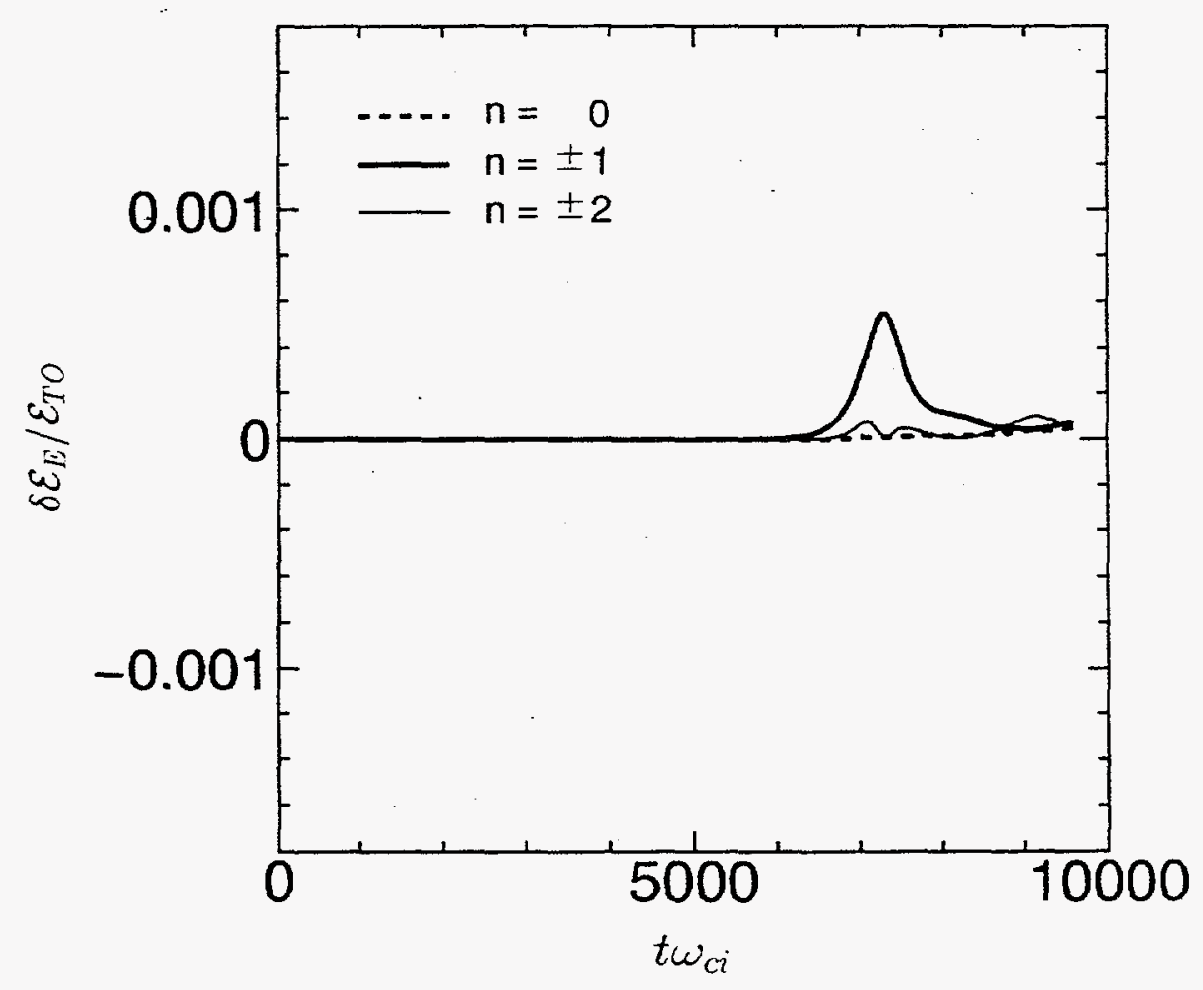

Fig. 12 
(I)

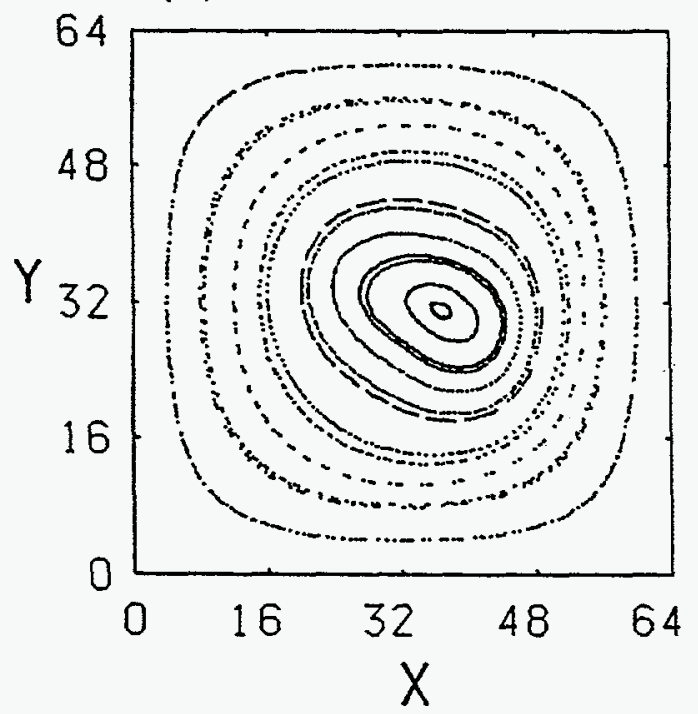

(II)

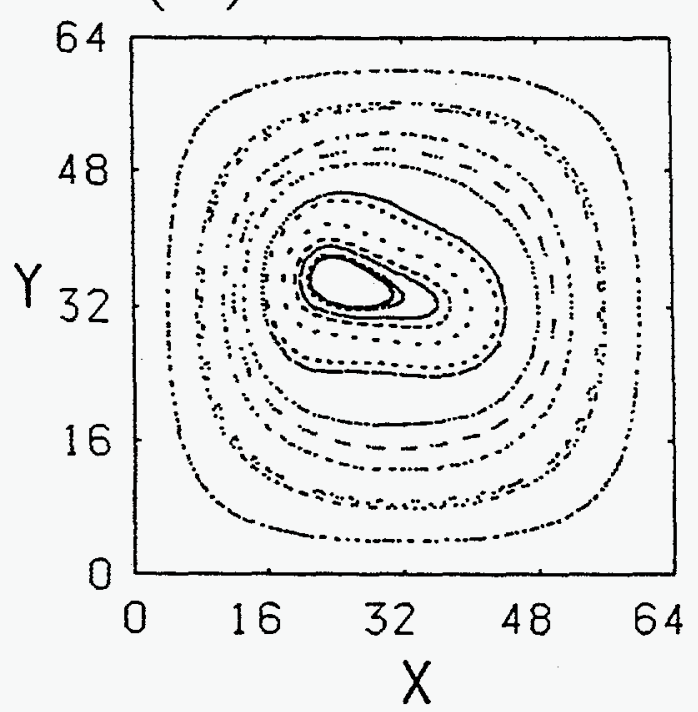

(III)

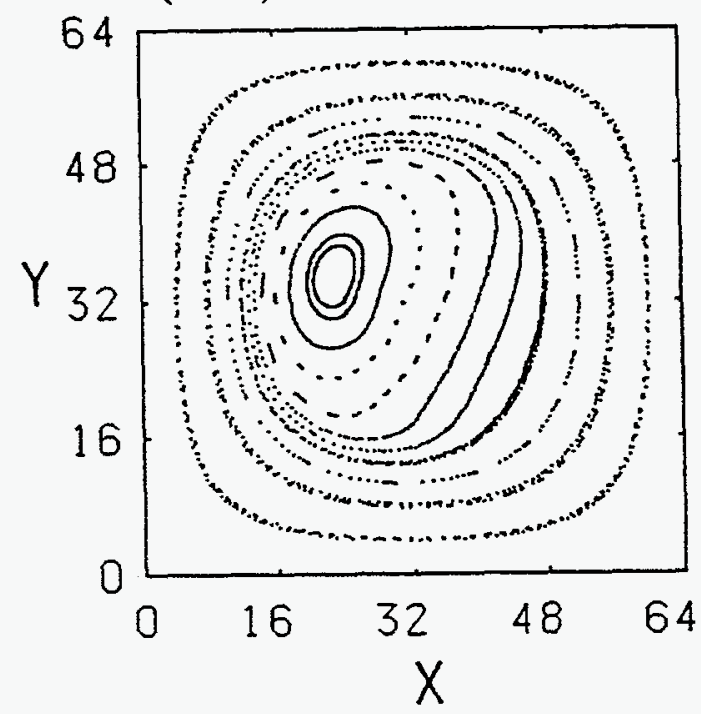

(IV)

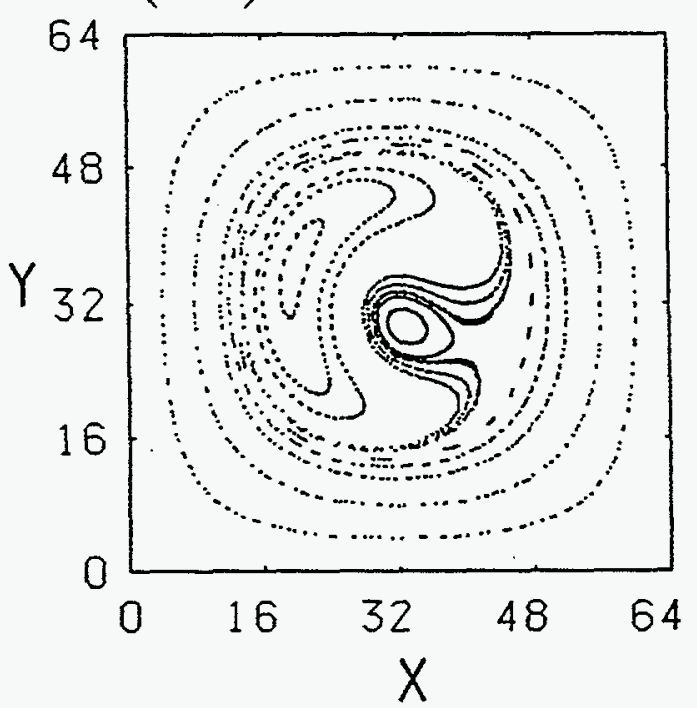

Fig. 13 
(I)

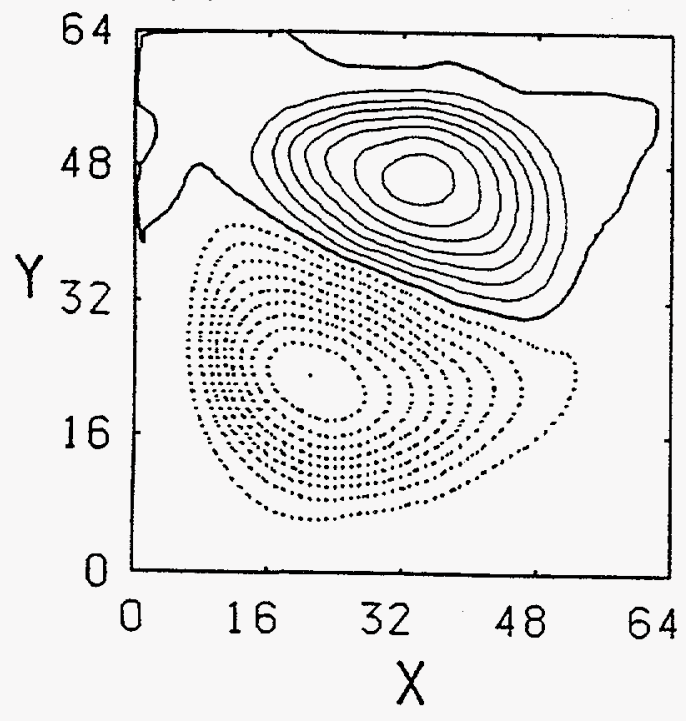

(II)

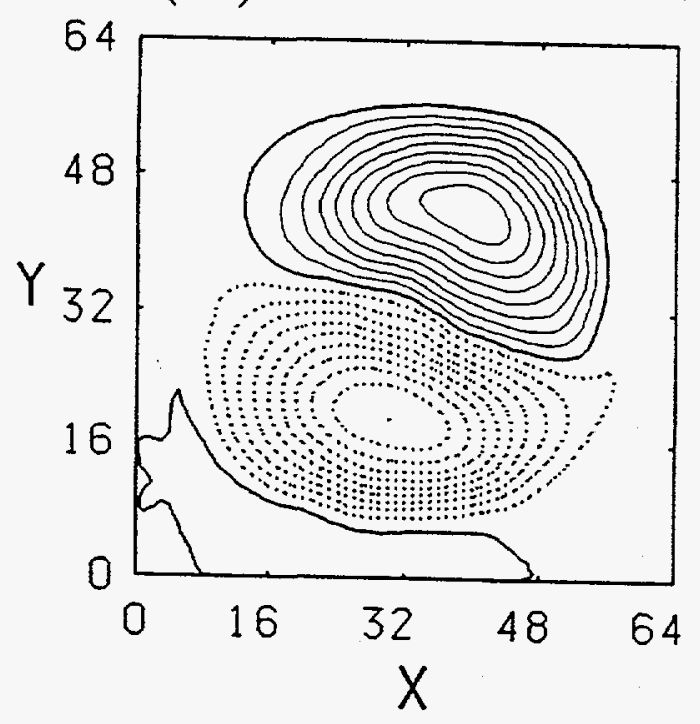

(III)

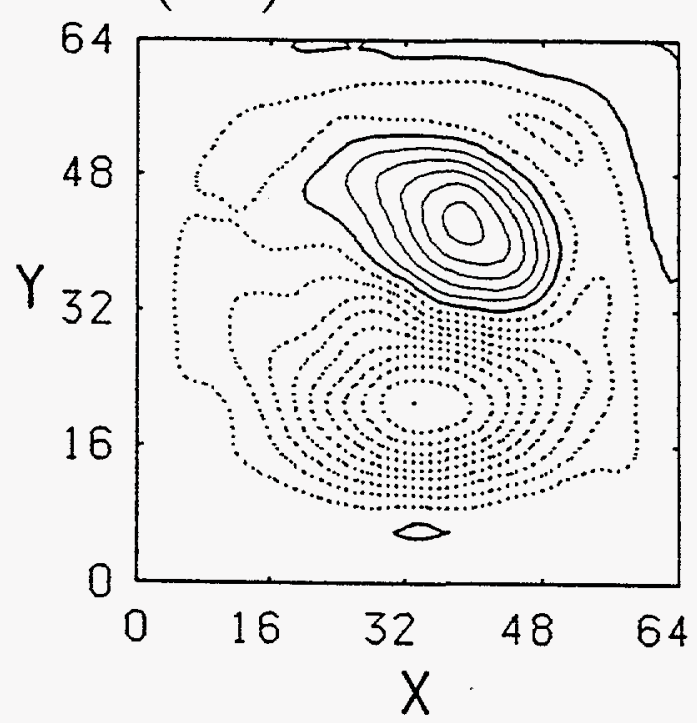

(IV)

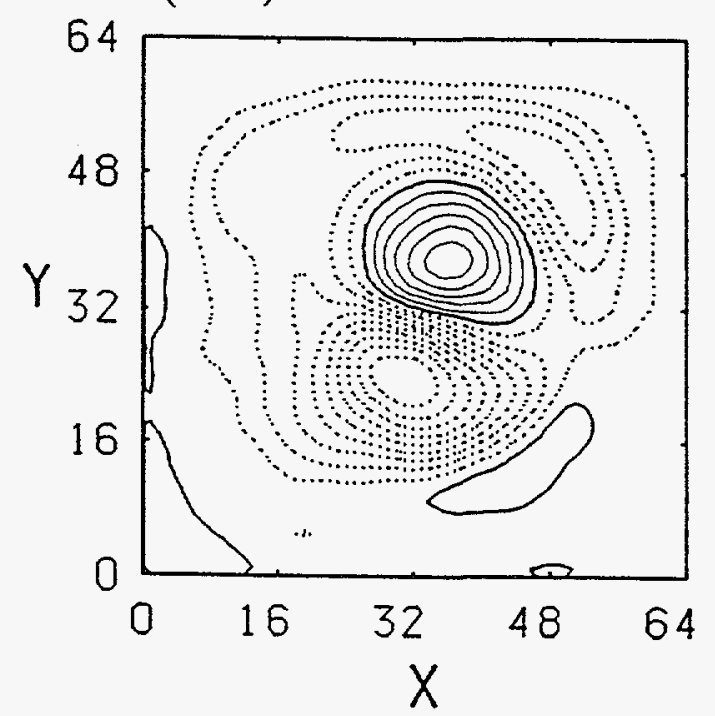

Fig. 14 
Dr. F. Paoloni, Univ. of Wollongong, AUSTRALIA

Prof. R.C. Cross, Univ. of Sydney, AUSTRALIA

Plasma Research Lab., Australian Nat. Univ., AUSTRALIA

Prof. I.R. Jones, Flinders Univ, AUSTRALIA

Prof. F. Cap, Inst. for Theoretical Physics, AUSTRIA

Prof. M. Heindler, Institut für Theoretische Physik, AUSTRIA

Prof. M. Goossens, Astronomisch Instituut, BELGIUM

Ecole Royale Militaire, Lab. de Phy. Plasmas, BELGIUM

Commission-European, DG. XII-Fusion Prog., BELGIUM

Prof. R. Boucique, Rijksuniversiteit Gent, BELGIUM

Dr. P.H. Sakanaka, Instituto Fisica, BRAZIL

Prof. Dr. I.C. Nascimento, Instituto Fisica, Sao Paulo, BRAZIL Instituto Nacional De Pesquisas Espaciais-INPE, BRAZIL Documents Office, Atomic Energy of Canada Ltd., CANADA Ms. M. Morin, CCFM/Tokamak de Varennes, CANADA Dr. M.P. Bachynski, MPB Technologies, Inc., CANADA

Dr. H.M. Skarsgard, Univ. of Saskatchewan, CANADA

Prof. J. Teichmann, Univ. of Montreal, CANADA

Prof. S.R. Sreenivasan, Univ. of Calgary, CANADA

Prof. R. Marchand, INRS-Energie et Materiaux, CANADA

Dr. R. Bolton, Centre canadien de fusion magnétique, CANADA

Dr. C.R. James, Univ. of Alberta, CANADA

Dr. P. Lukác, Komenského Universzita, CZECHO-SLOVAKIA

The Librarian, Culham Laboratory, ENGLAND

Library, R61, Rutherford Appleton Laboratory, ENGLAND

Mrs. S.A. Hutchinson, JET Library, ENGLAND

Dr. S.C. Sharma, Univ. of South Pacific, FIJI ISLANDS

P. Mähönen, Univ. of Helsinki, FINLAND

Prof. M.N. Bussac, Ecole Polytechnique,, FRANCE

C. Mouttet, Lab. de Physique des Milieux lonisés, FRANCE

J. Radet, CENCADARACHE - Bat 506, FRANCE

Prof. E. Economou, Univ. of Crete, GREECE

Ms. C. Rinni, Univ. of loannina, GREECE

Preprint Librany, Hungarian Academy of Sci., HUNGARY

Dr. B. DasGupta, Saha Inst. of Nuclear Physics, INDIA

Dr. P. Kaw, Inst. for Plasma Research, INDIA

Dr. P. Rosenau, Israel Inst. of Technology, ISRAEL Librarian, International Center for Theo Physics, ITALY Miss C. De Palo, Associazione EURATOM-ENEA, ITALY Dr. G. Grosso, Istituto di Fisica del Plasma, ITALY Prof. G. Rostangni, Istituto Gas lonizzati Del Cnr, ITALY
Dr. H. Yamato, Toshiba Res \& Devel Center, JAPAN

Prof. I. Kawakami, Hiroshima Univ., JAPAN

Prof. K. Nishikawa, Hiroshima Univ., JAPAN

Librarian, Naka Fusion Research Establishment, JAERI, JAPAN

Director, Japan Atomic Energy Research Inst., JAPAN

Prof. S. Itoh, Kyushu Univ., JAPAN

Research Info. Ctr., National Instit. for Fusion Science, JAPAN

Prof. S. Tanaka, Kyoto Univ., JAPAN

Library, Kyoto Univ., JAPAN

Prof. N. Inoue, Univ. of Tokyo, JAPAN

Secretary, Plasma Section, Electrotechnical Lab., JAPAN

Dr. O. Mitarai, Kumamoto Inst. of Technology, JAPAN

Dr. G.S. Lee, Korea Basic Sci. Ctr., KOREA

J. Hyeon-Sook, Korea Atomic Energy Research Inst., KOREA

D.I. Choi, The Korea Adv. Inst. of Sci. \& Tech., KOREA

Leandro Melendez Lugo, Inst. Nac'l. de Inves. Nucl, MEXICO

Prof. B.S. Liley, Univ. of Waikato, NEW ZEALAND

Inst of Physics, Chinese Acad Sci PEOPLE'S REP. OF CHINA

Library, Inst. of Plasma Physics, PEOPLE'S REP. OF CHINA

Tsinghua Univ. Library, PEOPLE'S REPUBLIC OF CHINA

Z. Li, S.W. Inst Physics, PEOPLE'S REPUBLIC OF CHINA

Prof. J.A.C. Cabral, Instituto Superior Tecnico, PORTUGAL

Prof. M.A. Hellberg, Univ. of Natal, S. AFRICA

Prof. D.E. Kim, Pohang inst. of Sci. \& Tech., SO. KOREA

Prof. C.I.E.M.A.T, Fusion Division Library, SPAIN

Dr. L. Stentlo, Univ. of UMEA, SWEDEN

Library, Royal Inst. of Technology, SWEDEN

Prof. H. Wilhelmson, Chalmers Univ. of Tech., SWEDEN

Centre Phys. Des Plasmas, Ecole Polytech, SWITZERLAND

Bibliotheek, Inst. Voor Plasma-Fysica, THE NETHERLANDS

Asst. Prof. Dr. S. Cakir, Middle East Tech. Univ., TURKEY

Dr. V.A. Glukhikh,Sci. Res. Inst. Electrophys.I Apparatus, USSR

Dr. D.D. Ryutov, Siberian Branch of Academy of Sai., USSR

Dr. G.A. Eliseev, I.V. Kurchatov Inst., USSR

Librarian, The Ukr.SSR Academy of Sciences, USSR

Dr. L.M. Kovrizhnykh, Inst. of General Physics, USSR

Kemforschungsanlage GmbH, Zentralbibliothek, W. GERMANY

Bibliothek, Inst. Für Plasmaforschung, W. GERMANY

Prof. K. Schindier, Ruhr-Universitát Bochum, W. GERMANY

Dr. F. Wagner, (ASDEX), Max-Planck-Institut, W. GERMANY

Librarian, Max-Planck-Institut, W. GERMANY 